\title{
Multidimensional Fixed-Point Theorems in Partially Ordered Complete Partial Metric Spaces under $(\psi, \varphi)$-Contractivity Conditions
}

\author{
A. Roldán, J. Martínez-Moreno, C. Roldán, and E. Karapınar \\ University of Jaén, Campus Las Lagunillas s/n, 23071 Jaén, Spain
}

Correspondence should be addressed to A. Roldán; afroldan@ujaen.es

Received 17 March 2013; Accepted 17 June 2013

Academic Editor: Abdelouahed Hamdi

Copyright (C) 2013 A. Roldán et al. This is an open access article distributed under the Creative Commons Attribution License, which permits unrestricted use, distribution, and reproduction in any medium, provided the original work is properly cited.

\begin{abstract}
We study the existence and uniqueness of coincidence point for nonlinear mappings of any number of arguments under a weak $(\psi, \varphi)$-contractivity condition in partial metric spaces. The results we obtain generalize, extend, and unify several classical and very recent related results in the literature in metric spaces (see Aydi et al. (2011), Berinde and Borcut (2011), Gnana Bhaskar and Lakshmikantham (2006), Berzig and Samet (2012), Borcut and Berinde (2012), Choudhury et al. (2011), Karapinar and Luong (2012), Lakshmikantham and Ćirić (2009), Luong and Thuan (2011), and Roldán et al. (2012)) and in partial metric spaces (see Shatanawi et al. (2012)).
\end{abstract}

\section{Introduction}

The notion of coupled fixed point was introduced by Guo and Lakshmikantham [1] in 1987. In a recent paper, Gnana Bhaskar and Lakshmikantham [2] introduced the concept mixed monotone property for contractive operators of the form $F: X \times X \rightarrow X$, where $X$ is a partially ordered metric space, and then established some coupled fixed-point theorems. After that, many results appeared on coupled fixedpoint theory in different contexts (see, e.g., [3-6]). Later, Berinde and Borcut [7] introduced the concept of tripled fixed point and proved tripled fixed-point theorems using mixed monotone mappings (see also [8-10]).

Very recently, Roldán et al. [11] proposed the notion of coincidence point between mappings in any number of variables and showed some existence and uniqueness theorems that extended the mentioned previous results for this kind of nonlinear mappings, not necessarily permuted or ordered, in the framework of partially ordered complete metric spaces, using a weaker contraction condition, that also generalized other works by Berzig and Samet [12], Karapinar and Berinde [13].

Partial metric spaces were firstly introduced by Matthews in [14] as an attempt to generalize the metric spaces by establishing the condition that the distance between a point to itself (which is not necessarily zero) is less or equal than the distance between that point and another point of the space. In the mentioned papers, Matthews studied topological properties of partial metric spaces and stated a modified version of a Banach contraction mapping principle on this kind of spaces. After Matthews' pioneering work, the theory of partial metric spaces and particularly the field of fixed-point theorems have expansively been developed due to the increasing interest in this area and motivated by its possible applications (see $[15,16]$ and references therein).

In this paper, our main aim is to study a weaker contractivity condition for nonlinear mappings of any number of arguments. This condition can be particularized in a variety of forms that let us extend the previously mentioned results and other recent ones in this field (see $[2,5,7,9,11,12,16-$ 20]). We also notice that our results cannot be obtained by the very recent paper of Haghi et al. [21] (for more details see Remark 26).

\section{Preliminaries}

Preliminaries and notation about coincidence points can also be found in [11]. Let $n$ be a positive integer. Henceforth, $X$ will denote a nonempty set, and $X^{n}$ will denote the product space 
$X^{n}=X \times X \times \stackrel{n}{ } . \times X$. Throughout this paper, $m$ and $k$ will denote nonnegative integers and $i, j, s \in\{1,2, \ldots, n\}$. Unless otherwise stated, "for all $m$ " will mean "for all $m \geq 0$ ", and "for all $i$ " will mean "for all $i \in\{1,2, \ldots, n\}$ ". Let $\mathbb{R}_{0}^{+}=[0, \infty[$.

A metric on $X$ is a mapping $d: X \times X \rightarrow \mathbb{R}$ satisfying, for all $x, y, z \in X$ :

(i) $d(x, y)=0$ if, and only if, $x=y$;

(ii) $d(x, y) \leq d(z, x)+d(z, y)$.

From these properties, we can easily deduce that $d(x, y) \geq$ 0 and $d(y, x)=d(x, y)$ for all $x, y \in X$. The last requirement is called the triangle inequality. If $d$ is a metric on $X$, we say that $(X, d)$ is a metric space (for short, an MS).

Definition 1 (see [22]). A triple $(X, d, \leq)$ is called a partially ordered metric space if $(X, d)$ is a MS and $\leq$ is a partial order on $X$.

Definition 2 (see [2]). An ordered MS $(X, d, \leq)$ is said to have the sequential $g$-monotone property if it verifies

(i) if $\left\{x_{m}\right\}$ is a nondecreasing sequence and $\left\{x_{m}\right\} \stackrel{d}{\rightarrow} x$, then $g x_{m} \leq g x$ for all $m$;

(ii) if $\left\{y_{m}\right\}$ is a nonincreasing sequence and $\left\{y_{m}\right\} \stackrel{d}{\rightarrow} y$, then $g y_{m} \geq g y$ for all $m$.

If $g$ is the identity mapping, then $X$ is said to have the sequential monotone property.

Henceforth, fix a partition $\{A, B\}$ of two non-empty subsets of $\Lambda_{n}=\{1,2, \ldots, n\}$; that is, $A \cup B=\Lambda_{n}$ and $A \cap B=\emptyset$. We will denote

$$
\begin{aligned}
& \Omega_{A, B}=\left\{\sigma: \Lambda_{n} \rightarrow \Lambda_{n}: \sigma(A) \subseteq A \text { and } \sigma(B) \subseteq B\right\}, \\
& \Omega_{A, B}^{\prime}=\left\{\sigma: \Lambda_{n} \rightarrow \Lambda_{n}: \sigma(A) \subseteq B \text { and } \sigma(B) \subseteq A\right\} .
\end{aligned}
$$

If $(X, \leq)$ is a partially ordered space, $x, y \in X$, and $i \in \Lambda_{n}$, we will use the following notation:

$$
x \leq_{i} y \Longleftrightarrow \begin{cases}x \leq y, & \text { if } i \in A, \\ x \geq y, & \text { if } i \in B .\end{cases}
$$

Let $F: X^{n} \rightarrow X$ and $g: X \rightarrow X$ be two mappings.

Definition 3 (see [11]). One says that $F$ and $g$ are commuting if $g F\left(x_{1}, \ldots, x_{n}\right)=F\left(g x_{1}, \ldots, g x_{n}\right)$ for all $x_{1}, \ldots, x_{n} \in X$.

Definition 4 (see [11]). Let $(X, \leq)$ be a partially ordered space. One says that $F$ has the mixed $g$-monotone property (with respect to $\{A, B\}$ ) if $F$ is $g$-monotone nondecreasing in arguments of $A$ and $g$-monotone nonincreasing in arguments of $B$; that is, for all $x_{1}, x_{2}, \ldots, x_{n}, y, z \in X$ and all $i$,

$$
\begin{aligned}
g y \leq & g z \\
& \Rightarrow F\left(x_{1}, \ldots, x_{i-1}, y, x_{i+1}, \ldots, x_{n}\right) \\
& \quad \leq_{i} F\left(x_{1}, \ldots, x_{i-1}, z, x_{i+1}, \ldots, x_{n}\right) .
\end{aligned}
$$

Henceforth, let $\sigma_{1}, \sigma_{2}, \ldots, \sigma_{n}, \tau: \Lambda_{n} \rightarrow \Lambda_{n}$ be $n+1$ mappings from $\Lambda_{n}$ into itself, and let $\Phi$ be the $(n+1)$-tuple $\left(\sigma_{1}, \sigma_{2}, \ldots, \sigma_{n}, \tau\right)$.
Definition 5 (see [11]). A point $\left(x_{1}, x_{2}, \ldots, x_{n}\right) \in X^{n}$ is called a $\Phi$-coincidence point of the mappings Fand $g$ if

$$
F\left(x_{\sigma_{i}(1)}, x_{\sigma_{i}(2)}, \ldots, x_{\sigma_{i}(n)}\right)=g x_{\tau(i)} \quad \forall i
$$

If $g$ is the identity mapping on $X$, then $\left(x_{1}, x_{2}, \ldots, x_{n}\right) \in X^{n}$ is called a $\Phi$-fixed point of the mapping $F$.

Remark 6. If $F$ and $g$ are commuting and $\left(x_{1}, x_{2}, \ldots, x_{n}\right) \in$ $X^{n}$ is a $\Phi$-coincidence point of $F$ and $g$, then $\left(g x_{1}\right.$, $\left.g x_{2}, \ldots, g x_{n}\right)$ also is a $\Phi$-coincidence point of $F$ and $g$.

Definition 7 (see [14]). A partial metric on $X$ is a mapping $p: X \times X \rightarrow \mathbb{R}_{0}^{+}$verifying, for all $x, y, z \in X:$

$$
\begin{aligned}
& \text { (P1) } p(x, x) \leq p(x, y) ; \\
& \text { (P2) } p(x, x)=p(x, y)=p(y, y) \Rightarrow x=y ; \\
& \text { (P3) } p(x, y)=p(y, x) ; \\
& \text { (P4) } p(x, z)+p(y, y) \leq p(x, y)+p(y, z) .
\end{aligned}
$$

In this case, $(X, p)$ is a partial metric space (for short, a PMS).

Example 8 (see, e.g., [14]). Let $X=\mathbb{R}_{0}^{+}$, and define $p$ on $X$ by $p(x, y)=\max \{x, y\}$ for all $x, y \in X$. Then, $(X, p)$ is a partial metric space.

Example 9 (see [14]). Let $X=\{[a, b]: a, b \in \mathbb{R}, a \leq b\}$, and define $p([a, b],[c, d])=\max \{b, d\}-\min \{a, c\}$. Then, $(X, p)$ is a partial metric space.

Example 10 (see [14]). Let $X=[0,1] \cup[2,3]$, and define $p$ : $X \times X \rightarrow \mathbb{R}_{0}^{+}$by

$$
p(x, y)= \begin{cases}\max \{x, y\}, & \text { if }\{x, y\} \cap[2,3] \neq \emptyset \\ |x-y|, & \text { if }\{x, y\} \subset[0,1]\end{cases}
$$

Then, $(X, p)$ is a partial metric space.

Example 11 (see, e.g., $[23,24])$. Let $(X, d)$ and $(X, p)$ be a metric space and a partial metric space, respectively. Functions $\rho_{i}: X \times X \rightarrow \mathbb{R}_{0}^{+}(i \in\{1,2,3\})$ given by

$$
\begin{aligned}
& \rho_{1}(x, y)=d(x, y)+p(x, y), \\
& \rho_{2}(x, y)=d(x, y)+\max \{u(x), u(y)\}, \\
& \rho_{3}(x, y)=d(x, y)+a,
\end{aligned}
$$

define partial metrics on $X$, where $u: X \rightarrow \mathbb{R}_{0}^{+}$is an arbitrary function and $a \geq 0$.

Obviously, if $(X, d)$ is a MS and we define $p=d$, then $(X, p)$ is a PMS. Indeed, a partial metric $p$ on $X$ verifies

(i) $p(x, y)=0 \Rightarrow x=y$;

(ii) $p(x, y)=p(y, x)$;

(iii) $p(x, z) \leq p(x, y)+p(y, z)$, 
but the condition $p(x, x)=0$ does not necessarily hold. For a partial metric $p$ on $X$, the mappings $d_{p}, d_{m}: X \times X \rightarrow \mathbb{R}_{0}^{+}$ given by

$$
\begin{gathered}
d_{p}(x, y)=2 p(x, y)-p(x, x)-p(y, y), \\
d_{m}(x, y)=\max \{p(x, y)-p(x, x), p(y, y)-p(y, y)\} \\
=p(x, y)-\min \{p(x, x), p(y, y)\},
\end{gathered}
$$

for all $x, y \in X$, are (usual) metrics on $X$. On a PMS, the concepts of convergence, Cauchy sequences, completeness, and continuity are defined as follows.

Definition 12 (see $[14,25,26])$. Let $\left\{x_{m}\right\}$ be a sequence on a $\operatorname{PMS}(X, p)$.

(i) $\left\{x_{m}\right\} p$-converges to $x \in X$ (and one will write $\left\{x_{m}\right\} \stackrel{p}{\rightarrow}$ $x)$ if $p(x, x)=\lim _{m \rightarrow \infty} p\left(x, x_{m}\right)$.

(ii) $\left\{x_{m}\right\}$ is called $p$-Cauchy if $\lim _{m, m^{\prime} \rightarrow \infty} p\left(x_{m}, x_{m^{\prime}}\right)$ exists (and it is finite).

(iii) $(X, p)$ is said to be $p$-complete if every $p$-Cauchy sequence $\left\{x_{m}\right\}$ in $X p$-converges to a point $x \in X$ such that $p(x, x)=\lim _{m, m^{\prime} \rightarrow \infty} p\left(x_{m}, x_{m^{\prime}}\right)$.

(iv) A mapping $f: X \rightarrow X$ is said to be $p$-continuous at $x_{0} \in X$ if, for every $\varepsilon>0$, there exists $\delta>0$ such that $f\left(B_{p}\left(x_{0}, \delta\right)\right) \subseteq B_{p}\left(f\left(x_{0}\right), \varepsilon\right)$.

We have used the previous notation because we need to distinguish between $p$-convergence and $d_{p}$-convergence on $X$ and usual convergence for real sequences.

Lemma 13 (see $[14,25,26])$. Let $\left\{x_{m}\right\}$ be a sequence on a PMS $(X, p)$.

(1) $\left\{x_{m}\right\}$ is p-Cauchy if, and only if, it is $d_{p}$-Cauchy.

(2) $\left\{x_{m}\right\} \stackrel{d_{p}}{\rightarrow} x$ if, and only if, $\left\{x_{m}\right\} \stackrel{p}{\rightarrow} x$ and $p(x, x)=$ $\lim _{m, m^{\prime} \rightarrow \infty} p\left(x_{m}, x_{m^{\prime}}\right)$; that is,

$$
\begin{aligned}
& \left\{d_{p}\left(x_{m}, x\right)\right\} \longrightarrow 0 \Longleftrightarrow p(x, x) \\
& \quad=\lim _{m \rightarrow \infty} p\left(x, x_{m}\right)=\lim _{m, m^{\prime} \rightarrow \infty} p\left(x_{m}, x_{m^{\prime}}\right) .
\end{aligned}
$$

(3) $(X, p)$ is complete if, and only if, the $M S\left(X, d_{p}\right)$ is complete.

(4) If $\left\{x_{m}\right\} \stackrel{p}{\rightarrow} x$ and $p(x, x)=0$, then $\lim _{m \rightarrow \infty} p\left(x_{m}, y\right)=$ $p(x, y)$ for all $y \in X$.

\section{Auxiliary Results}

We will use the following results about real sequences in the proof of our main theorems.

Lemma 14. Let $\left\{a_{m}^{1}\right\}_{m \in \mathbb{N}}, \ldots,\left\{a_{m}^{n}\right\}_{m \in \mathbb{N}}$ be $n$ real lower bounded sequences such that $\left\{\max \left(a_{m}^{1}, \ldots, a_{m}^{n}\right)\right\}_{m \in \mathbb{N}} \rightarrow \delta$. Then, there exists $i_{0} \in\{1,2, \ldots, n\}$ and a subsequence $\left\{a_{m(k)}^{i_{0}}\right\}_{k \in \mathbb{N}}$ such that $\left\{a_{m(k)}^{i_{0}}\right\}_{k \in \mathbb{N}} \rightarrow \delta$.
Proof. Let $b_{m}=\max \left(a_{m}^{1}, a_{m}^{2}, \ldots, a_{m}^{n}\right)$ for all $m$. As $\left\{b_{m}\right\}$ is convergent, it is bounded. As $a_{m}^{i} \leq b_{m}$ for all $m$ and $i$, then every $\left\{a_{m}^{i}\right\}$ is bounded. As $\left\{a_{m}^{1}\right\}_{m \in \mathbb{N}}$ is a real bounded sequence, it has a convergent subsequence $\left\{a_{\sigma_{1}(m)}^{1}\right\}_{m \in \mathbb{N}} \rightarrow a_{1}$. Consider the subsequences $\left\{a_{\sigma_{1}(m)}^{2}\right\}_{m \in \mathbb{N}},\left\{a_{\sigma_{1}(m)}^{3}\right\}_{m \in \mathbb{N}}, \ldots,\left\{a_{\sigma_{1}(m)}^{n}\right\}_{m \in \mathbb{N}}$; that are $n-1$ real bounded sequences and the sequence $\left\{b_{\sigma_{1}(m)}\right\}_{m \in \mathbb{N}}$ that also converges to $\delta$. As $\left\{a_{\sigma_{1}(m)}^{2}\right\}_{m \in \mathbb{N}}$ is a real bounded sequence, it has a convergent subsequence $\left\{a_{\sigma_{2} \sigma_{1}(m)}^{2}\right\}_{m \in \mathbb{N}} \rightarrow a_{2}$. Then, the sequences $\left\{a_{\sigma_{2} \sigma_{1}(m)}^{3}\right\}_{m \in \mathbb{N}}$, $\left\{a_{\sigma_{2} \sigma_{1}(m)}^{4}\right\}_{m \in \mathbb{N}}, \ldots,\left\{a_{\sigma_{2} \sigma_{1}(m)}^{n}\right\}_{m \in \mathbb{N}}$ also are $n-2$ real bounded sequences, $\left\{a_{\sigma_{2} \sigma_{1}(m)}^{1}\right\}_{m \in \mathbb{N}} \rightarrow a_{1}$, and $\left\{b_{\sigma_{2} \sigma_{1}(m)}\right\}_{m \in \mathbb{N}} \rightarrow \delta$. Repeating this process $n$ times, we can find $n$ subsequences $\left\{a_{\sigma(m)}^{1}\right\}_{m \in \mathbb{N}},\left\{a_{\sigma(m)}^{2}\right\}_{m \in \mathbb{N}}, \ldots,\left\{a_{\sigma(m)}^{n}\right\}_{m \in \mathbb{N}}$ (where $\left.\sigma=\sigma_{n} \cdots \sigma_{1}\right)$ such that $\left\{a_{\sigma(m)}^{i}\right\}_{m \in \mathbb{N}} \rightarrow a_{i}$ for all $i$. And $\left\{b_{\sigma(m)}\right\}_{m \in \mathbb{N}} \rightarrow \delta$. But

$$
\begin{aligned}
\left\{b_{\sigma(m)}\right\}_{m \in \mathbb{N}} & =\left\{\max \left(a_{\sigma(m)}^{n}, \ldots, a_{\sigma(m)}^{n}\right)\right\}_{m \in \mathbb{N}} \\
& \longrightarrow \max \left(a_{1}, \ldots, a_{n}\right)
\end{aligned}
$$

so $\delta=\max \left(a_{1}, \ldots, a_{n}\right)$, and there exists $i_{0} \in\{1,2, \ldots, n\}$ such that $a_{i_{0}}=\delta$. Therefore, there exists $i_{0} \in\{1,2, \ldots, n\}$ and a subsequence $\left\{a_{\sigma(m)}^{i_{0}}\right\}_{m \in \mathbb{N}}$ such that $\left\{a_{\sigma(m)}^{i_{0}}\right\}_{m \in \mathbb{N}} \rightarrow a_{i_{0}}=\delta$.

Lemma 15. Let $\left\{a_{m}\right\}_{m \in \mathbb{N}}$ be a sequence of nonnegative real numbers which has not any subsequence converging to zero. Then, for all $\varepsilon>0$, there exist $\delta \in] 0, \varepsilon\left[\right.$ and $m_{0} \in \mathbb{N}$ such that $a_{m} \geq \delta$ for all $m \geq m_{0}$.

Proof. Suppose that the conclusion is not true. Then, there exists $\varepsilon_{0}>0$ such that, for all $\left.\delta \in\right] 0, \varepsilon_{0}\left[\right.$, there exists $m_{0} \in \mathbb{N}$ verifying $a_{m_{0}}<\delta$. Let $k_{0} \in \mathbb{N}$ be such that $1 / k_{0}<\varepsilon_{0}$. For all $k \in \mathbb{N}$, take $\left.\delta_{k}=1 /\left(k+k_{0}\right) \in\right] 0, \varepsilon_{0}[$. Then, there exists $m(k) \in$ $\mathbb{N}$ verifying $0 \leq a_{m(k)}<\delta_{k}=1 /\left(k+k_{0}\right)$. Taking limit when $k \rightarrow \infty$, we deduce that $\lim _{k \rightarrow \infty} a_{m(k)}=0$. Then, $\left\{a_{m}\right\}$ has a subsequence converging to zero (maybe, reordering $\left\{a_{m(k)}\right\}$ ), but this is a contradiction.

Lemma 16. If $\left\{x_{m}\right\}_{m \in \mathbb{N}}$ is a sequence in a $M S(X, d)$ that is not Cauchy, then there exist $\varepsilon_{0}>0$ and two subsequences $\left\{x_{m(k)}\right\}_{k \in \mathbb{N}}$ and $\left\{x_{n(k)}\right\}_{k \in \mathbb{N}}$ such that, for all $k \in \mathbb{N}$,

$$
\begin{gathered}
k<m(k)<n(k)<m(k+1), \\
d\left(x_{m(k)}, x_{n(k)}\right) \geq \varepsilon_{0}, \quad d\left(x_{m(k)}, x_{n(k)-1}\right)<\varepsilon_{0} .
\end{gathered}
$$

Proof. We know that

$$
\begin{aligned}
& \left\{x_{m}\right\} \text { is Cauchy } \\
& \qquad \Longleftrightarrow\left[\forall \varepsilon>0, \exists n_{0} \in \mathbb{N}:\left(m, n \geq n_{0} \Longrightarrow d\left(x_{m}, x_{n}\right)<\varepsilon\right)\right] .
\end{aligned}
$$

If this condition is not true, then

$$
\exists \varepsilon_{0}>0:\left(\forall n_{0} \in \mathbb{N}, \exists m, n \geq n_{0} \text { such that } d\left(x_{m}, x_{n}\right) \geq \varepsilon_{0}\right)
$$


Let $n_{0}=2$. Then, there exists $m_{1}, n_{1} \in \mathbb{N}$ such that $m_{1}, n_{1} \geq n_{0}$ and $d\left(x_{m_{1}}, x_{n_{1}}\right) \geq \varepsilon_{0}$. Let $m(1)=\min \left(m_{1}, n_{1}\right) \geq n_{0}=2>1$, and consider the numbers

$$
\begin{gathered}
d\left(x_{m(1)}, x_{m(1)+1}\right), \\
d\left(x_{m(1)}, x_{m(1)+2}\right), \ldots, d\left(x_{m(1)}, x_{\max \left(m_{1}, n_{1}\right)}\right) .
\end{gathered}
$$

Since $d\left(x_{m(1)}, x_{\max \left(m_{1}, n_{1}\right)}\right)=d\left(x_{m_{1}}, x_{n_{1}}\right) \geq \varepsilon_{0}$, between the previous numbers there exists a first nonnegative integer $n(1) \in\left\{m(1)+1, m(1)+2, \ldots, \max \left(m_{1}, n_{1}\right)\right\}$ such that $d\left(x_{m(1)}, x_{n(1)}\right) \geq \varepsilon_{0}$ but $d\left(x_{m(1)}, x_{j}\right)<\varepsilon_{0}$ for all $j \in$ $\{m(1), m(1)+1, \ldots, n(1)-1\}$. In particular, $d\left(x_{m(1)}, x_{n(1)-1}\right)<$ $\varepsilon_{0}$.

Now, let $n_{0}=n(1)+1$. Then, there exists $m_{2}, n_{2} \in \mathbb{N}$ such that $m_{2}, n_{2} \geq n(1)+1$ and $d\left(x_{m_{2}}, x_{n_{2}}\right) \geq \varepsilon_{0}$. Let $m(2)=$ $\min \left(m_{2}, n_{2}\right) \geq n_{0}=n(1)+1>n(1)$, and consider the numbers

$$
\begin{gathered}
d\left(x_{m(2)}, x_{m(2)+1}\right), \\
d\left(x_{m(2)}, x_{m(2)+2}\right), \ldots, d\left(x_{m(2)}, x_{\max \left(m_{2}, n_{2}\right)}\right) .
\end{gathered}
$$

Since $d\left(x_{m(2)}, x_{\max \left(m_{2}, n_{2}\right)}\right)=d\left(x_{m_{2}}, x_{n_{2}}\right) \geq \varepsilon_{0}$, between the previous numbers there exists a first nonnegative integer $n(2) \in\left\{m(2)+1, m(2)+2, \ldots, \max \left(m_{2}, n_{2}\right)\right\}$ such that $d\left(x_{m(2)}, x_{n(2)}\right) \geq \varepsilon_{0}$ but $d\left(x_{m(2)}, x_{j}\right)<\varepsilon_{0}$ for all $j \in$ $\{m(2), m(2)+1, \ldots, n(2)-1\}$. In particular, $d\left(x_{m(2)}, x_{n(2)-1}\right)<$ $\varepsilon_{0}$.

Repeating this process, we can find two subsequences $\left\{x_{m(k)}\right\}$ and $\left\{x_{n(k)}\right\}$ such that, for all $k \in \mathbb{N}$ :

$$
\begin{gathered}
k<m(k)<n(k)<m(k+1), \\
d\left(x_{m(k)}, x_{n(k)}\right) \geq \varepsilon_{0}, \quad d\left(x_{m(k)}, x_{n(k)-1}\right)<\varepsilon_{0} .
\end{gathered}
$$

Definition 17. Let $\Psi$ be the family of all continuous, nondecreasing mappings $\psi: \mathbb{R}_{0}^{+} \rightarrow \mathbb{R}_{0}^{+}$such that $\psi(t)=0$ if, and only if, $t=0$.

These mappings are known as altering distance functions (see [27]). Note that every selected $\psi \in \Psi$ commutes with max; that is, $\psi\left(\max \left(s_{1}, s_{2}, \ldots, s_{N}\right)\right)=$ $\max \left(\psi\left(s_{1}\right), \psi\left(s_{2}\right), \ldots, \psi\left(s_{N}\right)\right)$ for all $s_{1}, s_{2}, \ldots, s_{N} \in[0, \infty)$.

Lemma 18. If $\psi \in \Psi$ and $\lim _{m \rightarrow \infty} \psi\left(a_{m}\right)=0$, then $\lim _{m \rightarrow \infty} a_{m}=0$.

Proof. As there exists $\psi\left(a_{m}\right)$, then $a_{m} \in \operatorname{dom} \psi=[0, \infty[$. If the conclusion is not true, there exists $\varepsilon_{0}>0$ such that, for all $n_{0} \in \mathbb{N}$, there exists $n \geq n_{0}$ verifying $a_{n} \geq \varepsilon_{0}$. This means that $\left\{a_{m}\right\}$ has a subsequence $\left\{a_{m(k)}\right\}_{k}$ such that $a_{m(k)} \geq \varepsilon_{0}$. As $\psi$ is nondecreasing, $\psi\left(\varepsilon_{0}\right) \leq \psi\left(a_{m(k)}\right)$ for all $k \in \mathbb{N}$. Therefore, $\left\{\psi\left(a_{m}\right)\right\}_{m}$ has a subsequence $\left\{\psi\left(a_{m(k)}\right)\right\}_{k}$ lower bounded by $\psi\left(\varepsilon_{0}\right)>0$, but this is impossible since $\lim _{m \rightarrow \infty} \psi\left(a_{m}\right)=0$.

With regards to coincidence points, it is possible to consider the following simplification. If $\tau$ is a permutation of $\Lambda_{n}$, and we reorder (4), then we deduce that every coincidence point may be seen as a coincidence point associated to the identity mapping on $\Lambda_{n}$ (see, for instance, [28]).

Lemma 19. Let $\tau$ be a permutation of $\Lambda_{n}$, and let $\Phi=$ $\left(\sigma_{1}, \sigma_{2}, \ldots, \sigma_{n}, \tau\right)$ and $\Phi^{\prime}=\left(\sigma_{\tau^{-1}(1)}, \sigma_{\tau^{-1}(2)}, \ldots, \sigma_{\tau^{-1}(n)}, I_{\Lambda_{n}}\right)$. Then, a point $\left(x_{1}, x_{2}, \ldots, x_{n}\right) \in X^{n}$ is a $\Phi$-coincidence point of the mappings $F$ and $g$ if, and only if, $\left(x_{1}, x_{2}, \ldots, x_{n}\right)$ is a $\Phi^{\prime}$ coincidence point of the mappings $F$ and $g$.

Therefore, in the sequel, without loss of generality, we will only consider $\Upsilon$-coincidence points where $\Upsilon=$ $\left(\sigma_{1}, \sigma_{2}, \ldots, \sigma_{n}\right)$, that is, that verify $F\left(x_{\sigma_{i}(1)}, x_{\sigma_{i}(2)}, \ldots, x_{\sigma_{i}(n)}\right)=$ $g x_{i}$ for all $i$. We also show some preliminary results on PMS.

Lemma 20. Let $\left\{x_{m}\right\}$ be a sequence on a PMS $(X, p)$, and let $x \in X$.

(1) If $\left\{x_{m}\right\} \underset{p}{\rightarrow} x$ and $p(x, x)=0$, then $\left\{x_{m}\right\} \stackrel{d_{p}}{\rightarrow} x$,
$\left\{d_{p}\left(x_{m}, y\right)\right\} \rightarrow d_{p}(x, y)$ and $\left\{p\left(x_{m}, y\right)\right\} \rightarrow p(x, y)$
for all $y \in X$. (2) If $\left\{x_{m}\right\} \stackrel{d_{p}}{\longrightarrow} x$ and $\left\{p\left(x_{m}, x_{m}\right)\right\} \rightarrow 0$, then $p(x, x)=0$.

Proof. (1) Since $0 \leq p\left(x_{m}, x_{m}\right) \leq p\left(x, x_{m}\right)$ and $\lim _{m \rightarrow \infty} p\left(x, x_{m}\right)=p(x, x)=0$, then $\lim _{m \rightarrow \infty} p\left(x_{m}, x_{m}\right)$ $=0$. Therefore, $\lim _{m \rightarrow \infty} d_{p}\left(x, x_{m}\right)=\lim _{m \rightarrow \infty}\left(2 p\left(x, x_{m}\right)-\right.$ $\left.p(x, x)-p\left(x_{m}, x_{m}\right)\right)=0$, so $\left\{x_{m}\right\} \stackrel{d_{p}}{\longrightarrow} x$. Since $d_{p}$ is continuous, then $\left\{d_{p}\left(x_{m}, y\right)\right\} \rightarrow d_{p}(x, y)$ for all $y \in X$, and item 4 of Lemma 13 implies that $\left\{p\left(x_{m}, y\right)\right\} \rightarrow p(x, y)$.

(2) Item 2 of Lemma 13 shows that $p(x, x)=$ $\lim _{m, m^{\prime} \rightarrow \infty} p\left(x_{m}, x_{m^{\prime}}\right)=\lim _{m \rightarrow \infty} p\left(x_{m}, x_{m}\right)=0$.

Remark 21. Although the limit in a MS is unique, the $p$-limit in a PMS is not necessarily unique. For instance, let $(X, p)$ as in Example 10. Then, $(X, p)$ is a complete PMS (see [14]). Consider $x_{m}=2.5-1 /(2 m)$ for all $m \in \mathbb{N}$. Then, $\left\{x_{m}\right\} \stackrel{d_{p}}{\longrightarrow} 2.5$ but $\left\{x_{m}\right\} \stackrel{p}{\rightarrow} x_{0}$ whenever $x_{0} \in[2.5,3]$.

Definition 22. Let $N \in \mathbb{N}$, let $(X, p)$ be a PMS, let $G: X^{N} \rightarrow$ $X$ be a mapping, and let $Y_{0}=\left(x_{1}, x_{2}, \ldots, x_{N}\right) \in X^{N}$. We will say that $G$ is $\alpha_{p}$-continuous at $Y_{0}$ if, for all sequences $\left\{x_{m}^{1}\right\},\left\{x_{m}^{2}\right\}, \ldots,\left\{x_{m}^{N}\right\}$ on $X$ such that $\left\{x_{m}^{i}\right\} \stackrel{p}{\rightarrow} x_{i}$ for all $i \epsilon$ $\{1,2, \ldots, N\}, p\left(x_{i}, x_{i}\right)=0$ for all $i \in\{1,2, \ldots, N\}$ and $\left\{p\left(G\left(x_{m}^{1}, x_{m}^{2}, \ldots, x_{m}^{N}\right), G\left(x_{m}^{1}, x_{m}^{2}, \ldots, x_{m}^{N}\right)\right)\right\} \rightarrow 0$, we have that $\left\{G\left(x_{m}^{1}, x_{m}^{2}, \ldots, x_{m}^{N}\right)\right\} \stackrel{p}{\rightarrow} G\left(Y_{0}\right)$ and $p\left(G\left(Y_{0}\right), G\left(Y_{0}\right)\right)=0$. One will say that $G$ is $\alpha_{p}$-continuous if it is continuous at every point $Y_{0} \in X^{N}$.

Lemma 23. If $(X, p)$ is a PMS, and $G: X^{N} \rightarrow X$ is $d_{p^{-}}$ continuous at $Y_{0} \in X^{N}$, then $G$ is $\alpha_{p}$-continuous at $Y_{0}$.

Proof. Let $\left\{x_{m}^{1}\right\},\left\{x_{m}^{2}\right\}, \ldots,\left\{x_{m}^{N}\right\}$ sequences on $X$ such that $\left\{x_{m}^{i}\right\} \quad \stackrel{p}{\rightarrow} \quad x_{i}$ for all $i \in\{1,2, \ldots, N\}$, $p\left(x_{i}, x_{i}\right)=0$ for all $i \in\{1,2, \ldots, N\}$, and $\left\{p\left(G\left(x_{m}^{1}, x_{m}^{2}, \ldots, x_{m}^{N}\right), G\left(x_{m}^{1}, x_{m}^{2}, \ldots, x_{m}^{N}\right)\right)\right\} \rightarrow 0$. Item 1 
of Lemma 20 implies that $\left\{x_{m}^{i}\right\} \stackrel{d_{p}}{\longrightarrow} x_{i}$ for all $i \in\{1,2, \ldots, N\}$. Since $G$ is $d_{p}$-continuous at $Y_{0}=\left(x_{1}, x_{2}, \ldots, x_{N}\right)$, then $\left\{G\left(x_{m}^{1}, x_{m}^{2}, \ldots, x_{m}^{N}\right)\right\} \quad \stackrel{d_{p}}{\longrightarrow} G\left(x_{1}, x_{2}, \ldots, x_{N}\right)$. Item 2 of Lemma 13 assures us that $\left\{G\left(x_{m}^{1}, \ldots, x_{m}^{N}\right)\right\} \stackrel{p}{\rightarrow} G\left(x_{1}, \ldots, x_{N}\right)$ and

$$
\begin{aligned}
p & \left(G\left(x_{1}, x_{2}, \ldots, x_{N}\right), G\left(x_{1}, x_{2}, \ldots, x_{N}\right)\right) \\
& =\lim _{m, m^{\prime} \rightarrow \infty} p\left(G\left(x_{m}^{1}, x_{m}^{2}, \ldots, x_{m}^{N}\right), G\left(x_{m^{\prime}}^{1}, x_{m^{\prime}}^{2}, \ldots, x_{m^{\prime}}^{N}\right)\right) \\
& =\lim _{m \rightarrow \infty} p\left(G\left(x_{m}^{1}, x_{m}^{2}, \ldots, x_{m}^{N}\right), G\left(x_{m}^{1}, x_{m}^{2}, \ldots, x_{m}^{N}\right)\right)=0 .
\end{aligned}
$$

Then, $G$ is $\alpha_{p}$-continuous at $Y_{0}$.

\section{Main Results}

In the following result, we show sufficient conditions to ensure the existence of $\Upsilon$-coincidence points, where $\Upsilon=$ $\left(\sigma_{1}, \sigma_{2}, \ldots, \sigma_{n}\right)$.

Theorem 24. Let $(X, p)$ be a complete PMS, and let $\leq$ a partial order on $X$. Let $\Upsilon=\left(\sigma_{1}, \sigma_{2}, \ldots, \sigma_{n}\right)$ be an $n$-tuple of mappings from $\{1,2, \ldots, n\}$ into itself verifying $\sigma_{i} \in \Omega_{A, B}$ if $i \in A$ and $\sigma_{i} \in \Omega_{A, B}^{\prime}$ if $i \in B$. Let $F: X^{n} \rightarrow X$ and $g: X \rightarrow X$ be two mappings such that $F$ has the mixed $g$-monotone property on $X, F\left(X^{n}\right) \subseteq g(X)$ and $g$ is $\alpha_{p}$-continuous and commuting with $F$. Assume that there exist $\psi, \varphi \in \Psi$ such that

$$
\begin{aligned}
& \psi\left(p\left(F\left(x_{1}, x_{2}, \ldots, x_{n}\right), F\left(y_{1}, y_{2}, \ldots, y_{n}\right)\right)\right) \\
& \quad \leq \psi\left(\max _{1 \leq i \leq n} p\left(g x_{i}, g y_{i}\right)\right)-\varphi\left(\max _{1 \leq i \leq n} p\left(g x_{i}, g y_{i}\right)\right),
\end{aligned}
$$

for which $g x_{i} \leq_{i} g y_{i}$ for all $i$. Suppose either $F$ is $\alpha_{p^{-}}$ continuous or $\left(X, d_{p}, \leq\right)$ has the sequential g-monotone property. If there exist $x_{0}^{1}, x_{0}^{2}, \ldots, x_{0}^{n} \in X$ verifying $g x_{0}^{i} \leq_{i} F\left(x_{0}^{\sigma_{i}(1)}, x_{0}^{\sigma_{i}(2)}, \ldots, x_{0}^{\sigma_{i}(n)}\right)$ for all $i$, then $F$ and $g$ have, at least, one $\Upsilon$-coincidence point.

Proof. The proof is divided into seven steps. The first two steps are the same as in the proof of Theorem 9 in [11], since the contractivity condition does not play any role in these parts of the proof.

Step 1. There exist $n$ sequences $\left\{x_{m}^{1}\right\}_{m \geq 0},\left\{x_{m}^{2}\right\}_{m \geq 0}, \ldots,\left\{x_{m}^{n}\right\}_{m \geq 0}$ such that $g x_{m+1}^{i}=F\left(x_{m}^{\sigma_{i}(1)}, x_{m}^{\sigma_{i}(2)}, \ldots, x_{m}^{\sigma_{i}(n)}\right)$ for all $m$ and all $i$.

Step 2. $g x_{m}^{i} \leq_{i} g x_{m+1}^{i}$ for all $m$ and all $i$.

Step 3. We claim that $\left\{p\left(g x_{m}^{i}, g x_{m+1}^{i}\right)\right\}_{m \geq 0} \rightarrow 0$ for all $i$ (i.e., $\left.\left\{\max _{1 \leq j \leq n} p\left(g x_{m}^{j}, g x_{m+1}^{j}\right)\right\}_{m \geq 0} \rightarrow 0\right)$.
Indeed, define $\delta_{m}=\max _{1 \leq j \leq n} p\left(g x_{m}^{j}, g x_{m+1}^{j}\right)$ for all $m$. As $g x_{m}^{i} \leq_{i} g x_{m+1}^{i}$ for all $m$ and all $i$, then condition (17) implies that, for all $m \geq 1$ and all $i$ :

$$
\begin{aligned}
& \psi\left(p\left(g x_{m}^{i}, g x_{m+1}^{i}\right)\right) \\
& =\psi\left(p\left(F\left(x_{m-1}^{\sigma_{i}(1)}, x_{m-1}^{\sigma_{i}(2)}, \ldots, x_{m-1}^{\sigma_{i}(n)}\right), F\left(x_{m}^{\sigma_{i}(1)}, x_{m}^{\sigma_{i}(2)}, \ldots, x_{m}^{\sigma_{i}(n)}\right)\right)\right) \\
& \leq \psi\left(\max _{1 \leq j \leq n} p\left(g x_{m-1}^{\sigma_{i}(j)}, g x_{m}^{\sigma_{i}(j)}\right)\right)-\varphi\left(\max _{1 \leq j \leq n} p\left(g x_{m-1}^{\sigma_{i}(j)}, g x_{m}^{\sigma_{i}(j)}\right)\right) \\
& \leq \psi\left(\max _{1 \leq j \leq n} p\left(g x_{m-1}^{j}, g x_{m}^{j}\right)\right)=\psi\left(\delta_{m-1}\right) .
\end{aligned}
$$

Therefore, for all $m \geq 1, \psi\left(\delta_{m}\right)=\psi\left(\max _{1 \leq i \leq n}\right.$ $\left.p\left(g x_{m}^{i}, g x_{m+1}^{i}\right)\right)=\max _{1 \leq i \leq n} \psi\left(p\left(g x_{m}^{i}, g x_{m+1}^{i}\right)\right) \leq \psi\left(\delta_{m-1}\right)$. This means that the sequence $\left\{\psi\left(\delta_{m}\right)\right\}_{m \geq 1}$ is nonincreasing and lower bounded. Hence, it is convergent; that is, there exists $\Delta \geq 0$ such that $\left\{\psi\left(\delta_{m}\right)\right\}_{m \geq 1} \rightarrow \Delta$. We are going to show that $\Delta=0$. Since

$$
\begin{aligned}
\left\{\max _{1 \leq i \leq n} \psi\right. & \left.\left(p\left(g x_{m}^{i}, g x_{m+1}^{i}\right)\right)\right\}_{m} \\
= & \left\{\psi\left(\max _{1 \leq i \leq n} p\left(g x_{m}^{i}, g x_{m+1}^{i}\right)\right)\right\}_{m} \\
= & \left\{\psi\left(\delta_{m}\right)\right\}_{m} \longrightarrow \Delta,
\end{aligned}
$$

Lemma 14 assures that there exist $i_{0} \in\{1,2, \ldots, n\}$ and a subsequence $\left\{\psi\left(p\left(g x_{m(k)}^{i_{0}}, g x_{m(k)+1}^{i_{0}}\right)\right)\right\}_{k}$ such that $\left\{\psi\left(p\left(g x_{m(k)}^{i_{0}}\right.\right.\right.$, $\left.\left.\left.g x_{m(k)+1}^{i_{0}}\right)\right)\right\}_{k} \rightarrow \Delta$. Repeating (18), for all $k \geq 1$,

$$
\begin{aligned}
\psi\left(p\left(g x_{m(k)}^{i_{0}}, g x_{m(k)+1}^{i_{0}}\right)\right) \\
\leq \psi \\
\psi\left(\max _{1 \leq j \leq n} p\left(g x_{m(k)-1}^{\sigma_{i_{0}(j)}}, g x_{m(k)}^{\sigma_{i_{0}}(j)}\right)\right) \\
\quad-\varphi\left(\max _{1 \leq j \leq n} p\left(g x_{m(k)-1}^{\sigma_{i_{0}}(j)}, g x_{m(k)}^{\sigma_{i_{0}}(j)}\right)\right) .
\end{aligned}
$$

Consider the sequence

$$
\left\{\max _{1 \leq j \leq n} p\left(g x_{m(k)-1}^{\sigma_{i_{0}}(j)}, g x_{m(k)}^{\sigma_{i_{0}}(j)}\right)\right\}_{k \geq 1} .
$$

Suppose that this sequence has no subsequence converging to zero. Using $\varepsilon=1$, Lemma 15 assures us that there exists $\delta^{\prime} \epsilon$ ]0, $1\left[\right.$ and $k_{0} \in \mathbb{N}$ such that $\max _{1 \leq j \leq n} p\left(g x_{m(k)-1}^{\sigma_{i_{0}}(j)}, g x_{m(k)}^{\sigma_{i_{0}}(j)}\right) \geq \delta^{\prime}$ for all $k \geq k_{0}$. It follows that

$$
-\varphi\left(\max _{1 \leq j \leq n} p\left(g x_{m(k)-1}^{\sigma_{i_{0}}(j)}, g x_{m(k)}^{\sigma_{i_{0}}(j)}\right)\right) \leq-\varphi\left(\delta^{\prime}\right) \quad \forall k \geq k_{0}
$$


Then, (20) says to us

$$
\begin{aligned}
\psi\left(p\left(g x_{m(k)}^{i_{0}}, g x_{m(k)+1}^{i_{0}}\right)\right) \\
\leq \psi\left(\max _{1 \leq j \leq n} p\left(g x_{m(k)-1}^{\sigma_{i_{0}}(j)}, g x_{m(k)}^{\sigma_{i_{0}}(j)}\right)\right) \\
\quad-\varphi\left(\max _{1 \leq j \leq n} p\left(g x_{m(k)-1}^{\sigma_{i_{0}}(j)}, g x_{m(k)}^{\sigma_{i_{0}}(j)}\right)\right) \\
\leq \psi\left(\max _{1 \leq j \leq n} p\left(g x_{m(k)-1}^{\sigma_{i_{0}}(j)}, g x_{m(k)}^{\sigma_{i_{0}}(j)}\right)\right)-\varphi\left(\delta^{\prime}\right) \\
\leq \psi\left(\delta_{m(k)-1}\right)-\varphi\left(\delta^{\prime}\right) .
\end{aligned}
$$

Taking limit in $k$, we deduce that $\Delta \leq \Delta-\varphi\left(\delta^{\prime}\right)<\Delta$, which is impossible. Therefore, the sequence in (21) must have a subsequence $\left\{\max _{1 \leq j \leq n} p\left(g x_{m^{\prime}(k)-1}^{\sigma_{i_{0}(j)}}, g x_{m^{\prime}(k)}^{\sigma_{i_{0}}(j)}\right)\right\}_{k \geq 1}$ converging to zero. Since $\psi$ and $\varphi$ are continuous, taking limit when $k \rightarrow \infty$ in (20) using this subsequence, we deduce that $0 \leq \Delta \leq \psi(0)-\varphi(0)=0$, so $\Delta=0$. Then, we have just proved that $\Delta=0$. Therefore, $\left\{\psi\left(\delta_{m}\right)\right\}_{m \geq 1} \rightarrow \Delta=0$, and Lemma 18 assures that $\left\{\delta_{m}\right\}_{m \geq 1} \rightarrow 0$, which means that $\left\{p\left(g x_{m}^{j}, g x_{m+1}^{j}\right)\right\} \rightarrow 0$ for all $j$ since $0 \leq p\left(g x_{m}^{j}, g x_{m+1}^{j}\right) \leq \delta_{m}$ for all $m$ and all $j$.

Step 4. $\left\{p\left(g x_{m}^{i}, g x_{m}^{i}\right)\right\}_{m \geq 0} \rightarrow 0$ for all $i$ (i.e., $\left\{\max _{1 \leq j \leq n}\right.$ $\left.\left.p\left(g x_{m}^{j}, g x_{m}^{j}\right)\right\}_{m \geq 0} \rightarrow 0\right)$. It is the same proof of Step 3 .

Since $d_{p}\left(g x_{m}^{i}, g x_{m+1}^{i}\right)=2 p\left(g x_{m}^{i}, g x_{m+1}^{i}\right)-p\left(g x_{m}^{i}, g x_{m}^{i}\right)-$ $p\left(g x_{m+1}^{i}, g x_{m+1}^{i}\right)$ for all $m$ and $i$, joining Steps 3 and 4 , it follows that

$$
\left\{d_{p}\left(g x_{m}^{i}, g x_{m+1}^{i}\right)\right\} \rightarrow 0 \quad \forall i
$$

Step 5. Every sequence $\left\{g x_{m}^{i}\right\}_{m \geq 0}$ is $d_{p}$-Cauchy. We reason by contradiction. Suppose that $\left\{g x_{m}^{i_{1}}\right\}_{m \geq 0}, \ldots,\left\{g x_{m}^{i_{s}}\right\}_{m \geq 0}$ are not $d_{p}$-Cauchy $(s \geq 1)$ and $\left\{g x_{m}^{i_{s+1}}\right\}_{m \geq 0}, \ldots,\left\{g x_{m}^{i_{n}}\right\}_{m \geq 0}$ are $d_{p}$-Cauchy, being $\left\{i_{1}, \ldots, i_{n}\right\}=\{1, \ldots, n\}$. By Lemma 16, for all $r \in\{1,2, \ldots, s\}$, there exists $\varepsilon_{r}>0$ and subsequences $\left\{g x_{m_{r}(k)}^{i_{r}}\right\}_{k \in \mathbb{N}}$ and $\left\{g x_{n_{r}(k)}^{i_{r}}\right\}_{k \in \mathbb{N}}$ such that

$$
\begin{gathered}
k<m_{r}(k)<n_{r}(k), \\
d_{p}\left(g x_{m_{r}(k)}^{i_{r}}, g x_{n_{r}(k)}^{i_{r}}\right) \geq \varepsilon_{r}, \\
d_{p}\left(g x_{m_{r}(k)}^{i_{r}}, g x_{n_{r}(k)-1}^{i_{r}}\right)<\varepsilon_{r}, \quad \forall k \in \mathbb{N} .
\end{gathered}
$$

Now, let $\varepsilon_{0}=\max \left(\varepsilon_{1}, \ldots, \varepsilon_{s}\right)>0$ and $\varepsilon_{0}^{\prime}=\min \left(\varepsilon_{1}, \ldots, \varepsilon_{s}\right)>$ 0 . Since $\left\{g x_{m}^{i_{s+1}}\right\}_{m \geq 0}, \ldots,\left\{g x_{m}^{i_{n}}\right\}_{m \geq 0}$ are $d_{p}$-Cauchy, for all $j \in$ $\left\{i_{s+1}, \ldots, i_{n}\right\}$, there exists $n_{1}^{j} \in \mathbb{N}$ such that if $m, m^{\prime} \geq n_{1}^{j}$, then $d_{p}\left(g x_{m}^{j}, g x_{m^{\prime}}^{j}\right)<\varepsilon_{0}^{\prime} / 8$. Since $\left\{p\left(g x_{m}^{j}, g x_{m}^{j}\right)\right\} \rightarrow 0$ by Step 4, there exists $n_{2}^{j} \in \mathbb{N}$ such that if $m \geq n_{2}^{j}$, then $p\left(g x_{m}^{j}, g x_{m}^{j}\right)<$ $\varepsilon_{0}^{\prime} / 8$. Define $n_{0}=\max _{j \in\left\{i_{s+1}, \ldots, i_{n}\right\}}\left(n_{1}^{j}, n_{2}^{j}\right)$. If $m, m^{\prime} \geq n_{0}$, then

$$
\begin{aligned}
0 & \leq p\left(g x_{m}^{j}, g x_{m^{\prime}}^{j}\right) \\
& =\frac{d_{p}\left(g x_{m}^{j}, g x_{m^{\prime}}^{j}\right)+p\left(g x_{m}^{j}, g x_{m}^{j}\right)+p\left(g x_{m^{\prime}}^{j}, g x_{m^{\prime}}^{j}\right)}{2} \\
& <\frac{\varepsilon_{0}^{\prime} / 8+\varepsilon_{0}^{\prime} / 8+\varepsilon_{0}^{\prime} / 8}{2}=\frac{3 \varepsilon_{0}^{\prime}}{16}<\frac{\varepsilon_{0}^{\prime}}{4} .
\end{aligned}
$$

Therefore, we have proved that there exists $n_{0} \in \mathbb{N}$ such that if $m, m^{\prime} \geq n_{0}$, then

$$
\begin{array}{r}
d_{p}\left(g x_{m}^{j}, g x_{m^{\prime}}^{j}\right)<\frac{\varepsilon_{0}^{\prime}}{4}, \quad p\left(g x_{m}^{j}, g x_{m^{\prime}}^{j}\right)<\frac{\varepsilon_{0}^{\prime}}{4}, \\
\forall j \in\left\{i_{s+1}, \ldots, i_{n}\right\} .
\end{array}
$$

Next, let $q \in\{1,2, \ldots, s\}$ such that $\varepsilon_{q}=\varepsilon_{0}=$ $\max \left(\varepsilon_{1}, \ldots, \varepsilon_{s}\right)$. Let $k_{1} \in \mathbb{N}$ such that $n_{0}<m_{q}^{\prime}\left(k_{1}\right)$, and define $m(1)=m_{q}\left(k_{1}\right)$. Consider the numbers $m(1)+1, m(1)+$ $2, \ldots, n_{q}\left(k_{1}\right)$ until finding the first positive integer $n(1)>$ $m(1)$ verifying

$$
\begin{array}{r}
\max _{1 \leq r \leq s} d_{p}\left(g x_{m(1)}^{i_{r}}, g x_{n(1)}^{i_{r}}\right) \geq \varepsilon_{0}, \quad d_{p}\left(g x_{m(1)}^{i_{j}}, g x_{n(1)-1}^{i_{j}}\right)<\varepsilon_{0}, \\
\forall j \in\{1,2, \ldots, s\} .
\end{array}
$$

Now let $k_{2} \in \mathbb{N}$ such that $n(1)<m_{q}\left(k_{2}\right)$, and define $m(2)=$ $m_{q}\left(k_{2}\right)$. Consider the numbers $m(2)+1, m(2)+2, \ldots, n_{q}\left(k_{2}\right)$ until finding the first positive integer $n(2)>m(2)$ verifying

$$
\begin{array}{r}
\max _{1 \leq r \leq s} d_{p}\left(g x_{m(2)}^{i_{r}}, g x_{n(2)}^{i_{r}}\right) \geq \varepsilon_{0}, \quad d_{p}\left(g x_{m(2)}^{i_{j}}, g x_{n(2)-1}^{i_{j}}\right)<\varepsilon_{0}, \\
\forall j \in\{1,2, \ldots, s\} .
\end{array}
$$

Repeating this process, we can find sequences such that, for all $k \geq 1$,

$$
\begin{gathered}
n_{0}<m(k)<n(k)<m(k+1), \\
\max _{1 \leq r \leq s} d_{p}\left(g x_{m(k)}^{i_{r}}, g x_{n(k)}^{i_{r}}\right) \geq \varepsilon_{0}, \\
d_{p}\left(g x_{m(k)}^{i_{j}}, g x_{n(k)-1}^{i_{j}}\right)<\varepsilon_{0}, \quad \forall j \in\{1,2, \ldots, s\} .
\end{gathered}
$$

Note that by (27), $d_{p}\left(g x_{m(k)}^{i_{r}}, g x_{n(k)}^{i_{r}}\right), d_{p}\left(g x_{m(k)}^{i_{r}}, g x_{n(k)-1}^{i_{r}}\right)<$ $\varepsilon_{0}^{\prime} / 4<\varepsilon_{0} / 2$ for all $r \in\{s+1, s+2, \ldots, n\}$, so

$$
\begin{gathered}
\max _{1 \leq j \leq n} d_{p}\left(g x_{m(k)}^{j}, g x_{n(k)}^{j}\right)=\max _{1 \leq r \leq s} d_{p}\left(g x_{m(k)}^{i_{r}}, g x_{n(k)}^{i_{r}}\right) \geq \varepsilon_{0}, \\
d_{p}\left(g x_{m(k)}^{i}, g x_{n(k)-1}^{i}\right)<\varepsilon_{0},
\end{gathered}
$$


for all $i \in\{1,2, \ldots, n\}$ and all $k \geq 1$. Furthermore, for all $j$,

$$
\begin{aligned}
& 2 p\left(g x_{m(k)-1}^{j}, g x_{n(k)-1}^{j}\right)-p\left(g x_{m(k)-1}^{j}, g x_{m(k)-1}^{j}\right) \\
& -p\left(g x_{n(k)-1}^{j}, g x_{n(k)-1}^{j}\right) \\
& \quad=d_{p}\left(g x_{m(k)-1}^{j}, g x_{n(k)-1}^{j}\right) \\
& \quad \leq d_{p}\left(g x_{m(k)-1}^{j}, g x_{m(k)}^{j}\right)+d_{p}\left(g x_{m(k)}^{j}, g x_{n(k)-1}^{j}\right) \\
& \quad \leq d_{p}\left(g x_{m(k)-1}^{j}, g x_{m(k)}^{j}\right)+\varepsilon_{0} .
\end{aligned}
$$

Therefore, for all $j$ and all $k$,

$$
\begin{aligned}
& p\left(g x_{m(k)-1}^{j}, g x_{n(k)-1}^{j}\right) \\
& \leq\left(\varepsilon_{0}+d_{p}\left(g x_{m(k)-1}^{j}, g x_{m(k)}^{j}\right)+p\left(g x_{m(k)-1}^{j}, g x_{m(k)-1}^{j}\right)\right. \\
& \left.\quad+p\left(g x_{n(k)-1}^{j}, g x_{n(k)-1}^{j}\right)\right) \times 2^{-1} .
\end{aligned}
$$

Next, for all $k$, let $i(k) \in\{1,2, \ldots, s\}$ be an index such that

$$
\begin{aligned}
d_{p}\left(g x_{m(k)}^{i(k)}, g x_{n(k)}^{i(k)}\right) & =\max _{1 \leq r \leq s} d_{p}\left(g x_{m(k)}^{i_{r}}, g x_{n(k)}^{i_{r}}\right) \\
& =\max _{1 \leq j \leq n} d_{p}\left(g x_{m(k)}^{j}, g x_{n(k)}^{j}\right) \geq \varepsilon_{0} .
\end{aligned}
$$

Then, for all $k$,

$$
\begin{gathered}
p\left(g x_{m(k)}^{i(k)}, g x_{n(k)}^{i(k)}\right) \\
=\left(d_{p}\left(g x_{m(k)}^{i(k)}, g x_{n(k)}^{i(k)}\right)+p\left(g x_{m(k)}^{i(k)}, g x_{m(k)}^{i(k)}\right)\right. \\
\left.\quad+p\left(g x_{n(k)}^{i(k)}, g x_{n(k)}^{i(k)}\right)\right) \times 2^{-1} \geq \frac{\varepsilon_{0}}{2} .
\end{gathered}
$$

Applying the contractivity condition (17), it follows, for all $k$,

$$
\begin{aligned}
0< & \psi\left(\frac{\varepsilon_{0}}{2}\right) \\
\leq & \psi\left(p\left(g x_{m(k)}^{i(k)}, g x_{n(k)}^{i(k)}\right)\right) \\
\leq & \psi\left(\max _{1 \leq j \leq n} p\left(g x_{m(k)-1}^{\sigma_{i(k)}(j)}, g x_{n(k)-1}^{\sigma_{i(k)}(j)}\right)\right) \\
& -\varphi\left(\max _{1 \leq j \leq n} p\left(g x_{m(k)-1}^{\sigma_{i(k)}(j)}, g x_{n(k)-1}^{\sigma_{i(k)}(j)}\right)\right) .
\end{aligned}
$$

Consider the sequence:

$$
\left\{\max _{1 \leq j \leq n} p\left(g x_{m(k)-1}^{\sigma_{i(k)}(j)}, g x_{n(k)-1}^{\sigma_{i(k)}(j)}\right)\right\}_{k \geq 1} .
$$

If this sequence has a subsequence that converges to zero, then we can take limit when $k \rightarrow \infty$ in (36) using this subsequence, so that we would have $0<\psi\left(\varepsilon_{0} / 2\right) \leq \psi(0)-$ $\varphi(0)=0$, which is impossible since $\varepsilon_{0}>0$. Therefore, the sequence (37) has no subsequence converging to zero. In this case, taking $\varepsilon_{0}>0$ in Lemma 15 , there exist $\left.\delta \in\right] 0, \varepsilon_{0}[$ and $k_{0} \in \mathbb{N}$ such that $\max _{1 \leq j \leq n} p\left(g x_{m(k)-1}^{\sigma_{i(k)}(j)}, g x_{n(k)-1}^{\sigma_{i(k)}(j)}\right) \geq$ $\delta$, for all $k \geq k_{0}$. It follows that, for all $k \geq k_{0}$, $-\varphi\left(\max _{1 \leq j \leq n} p\left(g x_{m(k)-1}^{\sigma_{i(k)}(j)}, g x_{n(k)-1}^{\sigma_{i(k)}(j)}\right)\right) \leq-\varphi(\delta)$. Thus, by (36),

$$
\begin{aligned}
0< & \psi\left(\frac{\varepsilon_{0}}{2}\right) \\
\leq & \psi\left(\max _{1 \leq j \leq n} p\left(g x_{m(k)-1}^{\sigma_{i(k)}(j)}, g x_{n(k)-1}^{\sigma_{i(k)}(j)}\right)\right) \\
& -\varphi\left(\max _{1 \leq j \leq n} p\left(g x_{m(k)-1}^{\sigma_{i(k)}(j)}, g x_{n(k)-1}^{\sigma_{i(k)}(j)}\right)\right) \\
\leq & \psi\left(\max _{1 \leq j \leq n} p\left(g x_{m(k)-1}^{\sigma_{i(k)}(j)}, g x_{n(k)-1}^{\sigma_{i(k)}(j)}\right)\right)-\varphi(\delta) \\
\leq & \psi\left(\max _{1 \leq j \leq n} p\left(g x_{m(k)-1}^{j}, g x_{n(k)-1}^{j}\right)\right)-\varphi(\delta) .
\end{aligned}
$$

Fix any $\gamma>0$ and we are going to prove that $\psi\left(\varepsilon_{0} / 2\right)+\varphi(\delta) \leq$ $\psi\left(\varepsilon_{0} / 2+\gamma\right)$. Indeed, by Step 3 and (24), since

$$
\begin{gathered}
\left\{\max _{1 \leq i \leq n} p\left(g x_{m(k)-1}^{i}, g x_{m(k)-1}^{i}\right)\right\}, \\
\left\{\max _{1 \leq i \leq n} p\left(g x_{n(k)-1}^{i}, g x_{n(k)-1}^{i}\right)\right\}, \\
\left\{\max _{1 \leq i \leq n} d_{p}\left(g x_{m(k)-1}^{i}, g x_{m(k)}^{i}\right)\right\}
\end{gathered}
$$

are sequences converging to zero, we can find $m_{1} \in \mathbb{N}$ such that if $m(k) \geq m_{1}$, then

$$
\begin{aligned}
& \max _{1 \leq i \leq n} p\left(g x_{m(k)-1}^{i}, g x_{m(k)-1}^{i}\right) \leq \frac{\gamma}{2}, \\
& \max _{1 \leq i \leq n} p\left(g x_{n(k)-1}^{i}, g x_{n(k)-1}^{i}\right) \leq \frac{\gamma}{2}, \\
& \max _{1 \leq i \leq n} d_{p}\left(g x_{m(k)-1}^{i}, g x_{m(k)}^{i}\right) \leq \frac{\gamma}{2} .
\end{aligned}
$$

Therefore, (33) implies that, for all $j$ and for all $k$ such that $m(k)>m_{1}$,

$$
\begin{gathered}
p\left(g x_{m(k)-1}^{j}, g x_{n(k)-1}^{j}\right) \\
\leq\left(\varepsilon_{0}+d_{p}\left(g x_{m(k)-1}^{j}, g x_{m(k)}^{j}\right)+p\left(g x_{m(k)-1}^{j}, g x_{m(k)-1}^{j}\right)\right. \\
\left.\quad+p\left(g x_{n(k)-1}^{j}, g x_{n(k)-1}^{j}\right)\right) \times 2^{-1} \\
\leq \frac{\varepsilon_{0}+\gamma / 2+\gamma / 2+\gamma / 2}{2}=\frac{\varepsilon_{0}}{2}+\frac{3 \gamma}{4}<\frac{\varepsilon_{0}}{2}+\gamma .
\end{gathered}
$$

Then, (38) guarantees that $0<\psi\left(\varepsilon_{0} / 2\right) \leq \psi\left(\max _{1 \leq j \leq n}\right.$ $\left.p\left(g x_{m(k)-1}^{j}, g x_{n(k)-1}^{j}\right)\right)-\varphi(\delta) \leq \psi\left(\varepsilon_{0} / 2+\gamma\right)-\varphi(\delta)$. This means that $\psi\left(\varepsilon_{0} / 2\right)+\varphi(\delta) \leq \psi\left(\varepsilon_{0} / 2+\gamma\right)$ for all $\gamma>0$. If we take $\gamma=1 / m>0$ (where $m \in \mathbb{N}$ ), we deduce that $\psi\left(\varepsilon_{0} / 2\right)+\varphi(\delta) \leq \psi\left(\varepsilon_{0} / 2+1 / m\right)$ for all $m \in \mathbb{N}$. Since $\psi$ is continuous, we have that $\psi\left(\varepsilon_{0} / 2\right)+\varphi(\delta) \leq \psi\left(\varepsilon_{0} / 2\right)$, which is 
impossible since $\varphi(\delta)>0$. This contradiction finally proves that every sequence $\left\{g x_{m}^{i}\right\}_{m \geq 0}$ is $d_{p}$-Cauchy.

Since $X$ is $p$-complete, then $X$ is $d_{p}$-complete (item 3 of Lemma 13). Then, there exist $x_{1}, x_{2}, \ldots, x_{n} \in X$ such that $\left\{g x_{m}^{i}\right\} \stackrel{d_{p}}{\longrightarrow} x_{i}$ for all $i$. Furthermore, $p\left(x_{i}, x_{i}\right)=$ $\lim _{m, m^{\prime} \rightarrow \infty} p\left(g x_{m}^{i}, g x_{m^{\prime}}^{i}\right)=\lim _{m \rightarrow \infty} p\left(g x_{m}^{i}, g x_{m}^{i}\right)=0$ for all $i$. Since $g$ is $\alpha_{p}$-continuous, then $\left\{g g x_{m}^{i}\right\} \stackrel{p}{\rightarrow} g x_{i}$ and $p\left(g x_{i}, g x_{i}\right)=0$ for all $i$. Item 1 of Lemma 20 shows that $\left\{g g x_{m}^{i}\right\} \stackrel{d_{p}}{\longrightarrow} g x_{i}$ for all $i$. Therefore, for all $i, \lim _{m \rightarrow \infty} p\left(g g x_{m+1}^{i}, g g x_{m+1}^{i}\right)=\lim _{m, m^{\prime} \rightarrow \infty}$ $p\left(g g x_{m+1}^{i}, g g x_{m^{\prime}+1}^{i}\right)=p\left(g x_{i}, g x_{i}\right)=0$. Moreover, for all $m$ and all $i, g g x_{m+1}^{i}=g F\left(x_{m}^{\sigma_{i}(1)}, x_{m}^{\sigma_{i}(2)}\right.$, $\left.\ldots, x_{m}^{\sigma_{n}(n)}\right)=F\left(g x_{m}^{\sigma_{i}(1)}, g x_{m}^{\sigma_{i}(2)}, \ldots, g x_{m}^{\sigma_{i}(n)}\right)$.

Step 6. Suppose that $F$ is $\alpha_{p}$-continuous. In this case, we know that $\left\{g x_{m}^{i}\right\} \stackrel{p}{\rightarrow} x_{i}$ and $p\left(x_{i}, x_{i}\right)=0$ for all $i$ and

$$
\begin{gathered}
\left\{p \left(F\left(g x_{m}^{\sigma_{i}(1)}, g x_{m}^{\sigma_{i}(2)}, \ldots, g x_{m}^{\sigma_{i}(n)}\right),\right.\right. \\
\left.\left.F\left(g x_{m}^{\sigma_{i}(1)}, g x_{m}^{\sigma_{i}(2)}, \ldots, g x_{m}^{\sigma_{i}(n)}\right)\right)\right\} \\
\quad=\left\{p\left(g g x_{m+1}^{i}, g g x_{m+1}^{i}\right)\right\} \longrightarrow 0,
\end{gathered}
$$

which implies that $\left\{F\left(g x_{m}^{\sigma_{i}(1)}, g x_{m}^{\sigma_{i}(2)}, \ldots, g x_{m}^{\sigma_{i}(n)}\right)\right\} \stackrel{p}{\rightarrow}$ $F\left(x_{\sigma_{i}(1)}, x_{\sigma_{i}(2)}, \ldots, x_{\sigma_{i}(n)}\right)$ and $p\left(F\left(x_{\sigma_{i}(1)}, \ldots, x_{\sigma_{i}(n)}\right)\right.$, $\left.F\left(x_{\sigma_{i}(1)}, \ldots, x_{\sigma_{i}(n)}\right)\right) \stackrel{0}{=} 0$ for all $i$. Item 1 of Lemma 20 assures us that, for all $i$,

$$
\begin{aligned}
\left\{g g x_{m+1}^{i}\right\} & =\left\{F\left(g x_{m}^{\sigma_{i}(1)}, g x_{m}^{\sigma_{i}(2)}, \ldots, g x_{m}^{\sigma_{i}(n)}\right)\right\} \\
& \stackrel{d_{p}}{\longrightarrow} F\left(x_{\sigma_{i}(1)}, x_{\sigma_{i}(2)}, \ldots, x_{\sigma_{i}(n)}\right) .
\end{aligned}
$$

Since the limit in a MS is unique, we deduce that $F\left(x_{\sigma_{i}(1)}, x_{\sigma_{i}(2)}, \ldots, x_{\sigma_{i}(n)}\right)=g x_{i}$ for all $i$, so $\left(x_{1}, x_{2}, \ldots, x_{n}\right)$ is a $Y$-coincidence point of $F$ and $g$.

Step 7. Suppose that $\left(X, d_{p}, \leq\right)$ has the sequential $g$-monotone property. In this case, by Step 2, we know that $g x_{m}^{i} \leq_{i} g x_{m+1}^{i}$ for all $m$ and all $i$. This means that the sequence $\left\{g x_{m}^{i}\right\}_{m \geq 0}$ is monotone. As $\left\{g x_{m}^{i}\right\} \stackrel{d_{p}}{\longrightarrow} x_{i}$, we deduce that $g g x_{m}^{i} \leq_{i} g x_{i}$ for all $m$ and all $i$. This condition implies that, for all $m$ and all j,

either $\left[g g^{\sigma_{j}^{(i)}} \leq_{i} g x_{\sigma_{j}(i)} \forall i\right]$ or $\left[g x_{\sigma_{j}(i)} \leq_{i} g g x_{m}^{\sigma_{j}^{(i)}} \forall i\right]$ (the first case occurs when $j \in A$ and the second one when $j \in B$ ). Then, by (17), for all $j$,

$$
\begin{aligned}
& \psi\left(p\left(g g x_{m+1}^{j}, F\left(x_{\sigma_{j}(1)}, x_{\sigma_{j}(2)}, \ldots, x_{\sigma_{j}(n)}\right)\right)\right) \\
& =\psi\left(p \left(F\left(g x_{m}^{\sigma_{j}(1)}, g x_{m}^{\sigma_{j}(2)}, \ldots, g x_{m}^{\sigma_{j}(n)}\right)\right.\right. \\
& \left.\left.F\left(x_{\sigma_{j}(1)}, x_{\sigma_{j}(2)}, \ldots, x_{\sigma_{j}(n)}\right)\right)\right) \\
& \leq \psi\left(\max _{1 \leq i \leq n} p\left(g g x_{m}^{\sigma_{j}^{(i)}}, g x_{\sigma_{j}(i)}\right)\right) \\
& -\varphi\left(\max _{1 \leq i \leq n} p\left(g g x_{m}^{\sigma_{j}(i)}, g x_{\sigma_{j}(i)}\right)\right) \\
& \leq \psi\left(\max _{1 \leq i \leq n} p\left(g g x_{m}^{i}, g x_{i}\right)\right) .
\end{aligned}
$$

Since $\left\{g g x_{m}^{i}\right\} \stackrel{d_{p}}{\longrightarrow} g x_{i}$ for all $i$, then

$$
\lim _{m \rightarrow \infty} p\left(g g x_{m}^{i}, g x_{i}\right)=p\left(g x_{i}, g x_{i}\right)=0 \quad \forall i .
$$

Therefore, $\lim _{m \rightarrow \infty}\left(\max _{1 \leq i \leq n} p\left(g g x_{m}^{i}, g x_{i}\right)\right)=0$. Taking limit when $m \rightarrow \infty$ in (45), we deduce that $\lim _{m \rightarrow \infty} \psi\left(p\left(g g x_{m+1}^{j}, F\left(x_{\sigma_{j}(1)}, x_{\sigma_{j}(2)}, \ldots, x_{\sigma_{j}(n)}\right)\right)\right)=0$ for all $j$. As $\psi \in \Psi$, Lemma 18 guarantees that

$$
\lim _{m \rightarrow \infty} p\left(g g x_{m+1}^{j}, F\left(x_{\sigma_{j}(1)}, x_{\sigma_{j}(2)}, \ldots, x_{\sigma_{j}(n)}\right)\right)=0 \quad \forall j .
$$

Finally, for all $j$,

$$
\begin{aligned}
& d_{p}\left(g x_{j}, F\left(x_{\sigma_{j}(1)}, x_{\sigma_{j}(2)}, \ldots, x_{\sigma_{j}(n)}\right)\right) \\
& =2 p\left(g x_{j}, F\left(x_{\sigma_{j}(1)}, x_{\sigma_{j}(2)}, \ldots, x_{\sigma_{j}(n)}\right)\right)-p\left(g x_{j}, g x_{j}\right) \\
& -p\left(F\left(x_{\sigma_{j}(1)}, x_{\sigma_{j}(2)}, \ldots, x_{\sigma_{j}(n)}\right)\right. \\
& \left.\quad F\left(x_{\sigma_{j}(1)}, x_{\sigma_{j}(2)}, \ldots, x_{\sigma_{j}(n)}\right)\right) \\
& \leq 2 p\left(g x_{j}, F\left(x_{\sigma_{j}(1)}, x_{\sigma_{j}(2)}, \ldots, x_{\sigma_{j}(n)}\right)\right) \\
& \leq 2\left[p\left(g g x_{m}^{i}, F\left(x_{\sigma_{j}(1)}, x_{\sigma_{j}(2)}, \ldots, x_{\sigma_{j}(n)}\right)\right) p\left(g x_{j}, g g x_{m}^{i}\right)\right. \\
& \left.\quad+p\left(g g x_{m}^{i}, F\left(x_{\sigma_{j}(1)}, x_{\sigma_{j}(2)}, \ldots, x_{\sigma_{j}(n)}\right)\right)\right] .
\end{aligned}
$$

Using (46) and (47), we conclude that $d_{p}\left(g x_{j}\right.$, $\left.F\left(x_{\sigma_{j}(1)}, x_{\sigma_{j}(2)}, \ldots, x_{\sigma_{j}(n)}\right)\right)=0$ for all $j$.

Remark 25. In the previous theorem, if the image Im $d$ of the metric $d$ is not the whole set $[0, \infty[$, then $\psi$ and $\varphi$ can only be defined on $\operatorname{Im} d$, and we can consider a wider range of mappings since it is only necessary to impose that they are continuous and nondecreasing on $\operatorname{Im} d$. 
Remark 26. We notice also that our paper cannot be deduced from the recent interesting paper of Haghi et al. [21] on partial metric space. In fact, we use a partial order $\leq$. Then, we only suppose (17) for which $g x_{i} \leq_{i} g y_{i}$ for all $i$ (not necessarily on points which are not comparable). Further, we use a self-map $g: X \rightarrow X$ which implies that

$$
\begin{gathered}
P(A, B)=\max _{1 \leq i \leq n} p\left(g a_{i}, g b_{i}\right), \\
A=\left(a_{1}, a_{2}, \ldots, a_{n}\right), \quad B=\left(b_{1}, b_{2}, \ldots, b_{n}\right) \in X^{n}
\end{gathered}
$$

is not necessarily a partial metric on $X^{n}$. For instance, let $X=$ $\mathbb{R}_{0}^{+}=[0, \infty)$ provided with its usual partial order and the partial metric $p(x, y)=\max (x, y)$. Consider

$$
g x= \begin{cases}0, & \text { if } 0 \leq x \leq 1, \\ x-1, & \text { if } x>1\end{cases}
$$

Then, $g$ is continuous, but

$$
\begin{aligned}
P & ((0,0, \ldots, 0),(0,0, \ldots, 0)) \\
& =P((1,1, \ldots, 1),(0,0, \ldots, 0)) \\
& =P((1,1, \ldots, 1),(1,1, \ldots, 1))=0,
\end{aligned}
$$

but $(0,0, \ldots, 0) \neq(1,1, \ldots, 1)$. Then, $P$ does not verify the axiom $p(x, x)=p(x, y)=p(y, y) \Rightarrow x=y$. Therefore, we cannot apply Theorem 2.4 on Haghi et al. [21].

As a result, we cannot use Theorem 2.7 in [21] since $T$ has an influence in $-\varphi(\max \{p(x, y), p(y, T y)\})$, and our mapping $F$ has not a role in the left side of (17).

\section{Consequences}

Remark 27. Theorem 9 in [11] is an easy consequence of Theorem 24 if we take $p=d, \psi(t)=t$, and $\varphi(t)=(1-k) t$ for all $t \in \mathbb{R}_{0}^{+}$.

In the next result, let $\Gamma_{0}$ be the family of all nondecreasing on each argument, continuous mappings $\phi:\left[0, \infty\left[{ }^{n} \rightarrow \mathbb{R}_{0}^{+}\right.\right.$ verifying $\phi\left(x_{1}, x_{2}, \ldots, x_{n}\right)=0$ if, and only if, $x_{1}=x_{2}=\cdots=$ $x_{n}=0$. Examples of such mappings are the following, where $k>0, \alpha_{i}>0$ and $n_{i} \in \mathbb{N}$ for all $i$.

(i) $\phi\left(x_{1}, \ldots, x_{n}\right)=k \max _{1 \leq i \leq n} x_{i}$.

(ii) $\phi\left(x_{1}, \ldots, x_{n}\right)=\sum_{i=1}^{n} \alpha_{i} x_{i}^{n_{i}}$.

(iii) $\phi\left(x_{1}, \ldots, x_{n}\right)=\sqrt[m]{\alpha_{1} x_{1}^{2}+\cdots+\alpha_{n} x_{n}^{2}}$.

Lemma 28. Let $\phi \in \Gamma_{0}$, and define $\varphi: \mathbb{R}_{0}^{+} \rightarrow \mathbb{R}_{0}^{+}$as $\varphi(t)=\min \left(\phi\left(t e_{1}\right), \phi\left(t e_{2}\right), \ldots, \phi\left(t e_{n}\right)\right)$ for all $t \geq 0$, where $\left\{e_{1}, e_{2}, \ldots, e_{n}\right\}$ is the usual basis of $\mathbb{R}^{n}$. Then, $\varphi \in \Psi$ and $\varphi\left(\max _{1 \leq i \leq n} x_{i}\right) \leq \phi\left(x_{1}, x_{2}, \ldots, x_{n}\right)$ for all $x_{1}, x_{2}, \ldots, x_{n} \in \mathbb{R}_{0}^{+}$.

Proof. First part is clear. If $x_{i_{0}}=\max _{1 \leq i \leq n} x_{i}$, then $\phi\left(x_{i_{0}} e_{i_{0}}\right)=\phi\left(0,0, \ldots, 0, x_{i_{0}}, 0, \ldots, 0\right) \leq \phi\left(x_{1}, x_{2}, \ldots\right.$, $\left.x_{i_{0}-1}, x_{i_{0}}, x_{i_{0}+1}, \ldots, x_{n}\right)=\phi\left(x_{1}, x_{2}, \ldots, x_{n}\right)$. Therefore, $\varphi\left(\max _{1 \leq i \leq n} x_{i}\right)=\varphi\left(x_{i_{0}}\right) \leq \phi\left(x_{i_{0}} e_{i_{0}}\right) \leq \phi\left(x_{1}, x_{2}, \ldots, x_{n}\right)$.

Corollary 29. Thesis of Theorem 24 also holds if one replaces the contractivity condition (17) by any of the following list (for which $g x_{i} \leq_{i} g y_{i}$ for all $\left.i\right)$.
(A) This condition can be found in [11] and [12], there exist $\psi \in \Psi$ and $\phi \in \Gamma_{0}$ such that

$$
\begin{aligned}
\psi(p & \left.\left(F\left(x_{1}, x_{2}, \ldots, x_{n}\right), F\left(y_{1}, y_{2}, \ldots, y_{n}\right)\right)\right) \\
\leq & \psi\left(\max _{1 \leq i \leq n} p\left(g x_{i}, g y_{i}\right)\right) \\
& -\phi\left(p\left(g x_{1}, g y_{1}\right), \ldots, p\left(g x_{n}, g y_{n}\right)\right) .
\end{aligned}
$$

(B) In [17], there exist $\psi, \varphi \in \Psi$ and $\beta_{1}, \beta_{2}, \ldots, \beta_{n} \in[0,1]$ such that $\beta_{1}+\beta_{2}+\cdots+\beta_{n} \leq 1$ and

$$
\begin{aligned}
& \psi\left(p\left(F\left(x_{1}, x_{2}, \ldots, x_{n}\right), p\left(y_{1}, y_{2}, \ldots, y_{n}\right)\right)\right) \\
& \quad \leq \psi\left(\sum_{i=1}^{n} \beta_{i} p\left(g x_{i}, g y_{i}\right)\right)-\varphi\left(\max _{1 \leq i \leq n} p\left(g x_{i}, g y_{i}\right)\right) .
\end{aligned}
$$

(C) There exist $\psi, \varphi \in \Psi$ and $\alpha_{1}, \alpha_{2}, \ldots, \alpha_{n}>0$ such that

$$
\begin{aligned}
& \psi\left(p\left(F\left(x_{1}, x_{2}, \ldots, x_{n}\right), F\left(y_{1}, y_{2}, \ldots, y_{n}\right)\right)\right) \\
& \quad \leq \psi\left(\max _{1 \leq i \leq n} p\left(g x_{i}, g y_{i}\right)\right)-\varphi\left(\sum_{i=1}^{n} \alpha_{i} p\left(g x_{i}, g y_{i}\right)\right) .
\end{aligned}
$$

(D) In $[2,7,9]$, there exist $\psi \in \Psi, \alpha_{1}, \alpha_{2}, \ldots, \alpha_{n}>0$, and $\beta_{1}, \beta_{2}, \ldots, \beta_{n} \geq 0$ such that $\beta_{1}+\beta_{2}+\cdots+\beta_{n} \leq 1$ and

$$
\begin{aligned}
& \psi\left(p\left(F\left(x_{1}, x_{2}, \ldots, x_{n}\right), F\left(y_{1}, y_{2}, \ldots, y_{n}\right)\right)\right) \\
& \quad \leq \psi\left(\sum_{i=1}^{n} \beta_{i} p\left(g x_{i}, g y_{i}\right)\right)-\sum_{i=1}^{n} \alpha_{i} p\left(g x_{i}, g y_{i}\right) .
\end{aligned}
$$

(E) In [5], there exist $\psi \in \Psi$ and $\alpha_{1}, \alpha_{2}, \ldots, \alpha_{n}>0$ such that

$$
\begin{aligned}
& \psi\left(p\left(F\left(x_{1}, x_{2}, \ldots, x_{n}\right), F\left(y_{1}, y_{2}, \ldots, y_{n}\right)\right)\right) \\
& \quad \leq \psi\left(\frac{1}{n} \sum_{i=1}^{n} p\left(g x_{i}, g y_{i}\right)\right)-\sum_{i=1}^{n} \alpha_{i} p\left(g x_{i}, g y_{i}\right) .
\end{aligned}
$$

(F) In $[19,20]$, there exist $\psi, \varphi \in \Psi$ such that $\psi$ is subadditive $(\psi(s+t) \leq \psi(s)+\psi(t)$ for all $t, s \in[0, \infty))$ and

$$
\begin{aligned}
\psi(p & \left.\left(F\left(x_{1}, x_{2}, \ldots, x_{n}\right), F\left(y_{1}, y_{2}, \ldots, y_{n}\right)\right)\right) \\
& \leq \frac{1}{n} \psi\left(\sum_{i=1}^{n} p\left(g x_{i}, g y_{i}\right)\right)-\varphi\left(\max _{1 \leq i \leq n} p\left(g x_{i}, g y_{i}\right)\right) .
\end{aligned}
$$

Of course, it is also interesting to particularize all the previous items to the following cases: $\psi(t)=\lambda t$ (where $\lambda>$ $0), \varphi(t)=\mu t$ (where $\mu>0)$, or $g x=x$ for all $x \in X$.

Proof. (A) By Lemma 28, there exists $\varphi \in \Psi$ such that $-\phi\left(x_{1}, x_{2}, \ldots, x_{n}\right) \leq-\varphi\left(\max _{1 \leq i \leq n} x_{i}\right)$ for all $x_{1}, x_{2}, \ldots, x_{n} \in[0, \infty[$, so (52) implies (17). (B) It is obvious that $\sum_{i=1}^{n} \beta_{i} p\left(g x_{i}, g y_{i}\right) \leq\left(\sum_{i=1}^{n} \beta_{i}\right) \max _{1 \leq j \leq n} p\left(g x_{j}, g y_{j}\right) \leq$ $\max _{1 \leq j \leq n} p\left(g x_{j}, g y_{j}\right)$, so (53) implies (17). (C) We only 
take $\phi\left(x_{1}, x_{2}, \ldots, x_{n}\right)=\varphi\left(\sum_{i=1}^{n} \alpha_{i} x_{i}\right)$ in item (A). (D) It is a mixture of (B) and (C). (E) It is a particular case of (D) where $\beta_{i}=1 / n$ for all $i$. (F) If $\psi$ is subadditive, then $(1 / n) \psi(t) \leq \psi(t / n)$ for all $t \geq 0$, so we may choose $\beta_{i}=1 / n$ for all $i$ in (B).

\section{Uniqueness of $\Upsilon$-Coincidence Points}

Consider on the product space $X^{n}$ the following partial order: for $\left(x_{1}, x_{2}, \ldots, x_{n}\right),\left(y_{1}, y_{2}, \ldots, y_{n}\right) \in X^{n}$,

$$
\left(x_{1}, x_{2}, \ldots, x_{n}\right) \leq\left(y_{1}, y_{2}, \ldots, y_{n}\right) \Longleftrightarrow x_{i} \leq_{i} y_{i}, \quad \forall i \text {. }
$$

We say that $\left(x_{1}, x_{2}, \ldots, x_{n}\right)$ and $\left(y_{1}, y_{2}, \ldots, y_{n}\right)$ are comparable if $\left(x_{1}, x_{2}, \ldots, x_{n}\right) \leq\left(y_{1}, y_{2}, \ldots, y_{n}\right)$ or $\left(x_{1}, x_{2}, \ldots, x_{n}\right) \geq$ $\left(y_{1}, y_{2}, \ldots, y_{n}\right)$.

Theorem 30. Under the hypothesis of Theorem 24, assume that for all $\Upsilon$-coincidence points $\left(x_{1}, x_{2}, \ldots, x_{n}\right)$, $\left(y_{1}, y_{2}, \ldots, y_{n}\right) \in X^{n}$ of $F$ and $g$ there exists $\left(u_{1}, u_{2}, \ldots, u_{n}\right) \in X^{n}$ such that $\left(g u_{1}, g u_{2}, \ldots, g u_{n}\right)$ is comparable, at the same time, to $\left(g x_{1}, g x_{2}, \ldots, g x_{n}\right)$ and to $\left(g y_{1}, g y_{2}, \ldots, g y_{n}\right)$.

Then, $F$ and $g$ have a unique $\Upsilon$-coincidence point $\left(z_{1}, z_{2}\right.$, $\left.\ldots, z_{n}\right) \in X^{n}$ such that $g z_{i}=z_{i}$ for all $i$.

Proof. From Theorem 24, the set of $\Upsilon$-coincidence points of $F$ and $g$ is nonempty. The proof is divided into two steps.

Step 1. We claim that if $\left(x_{1}, x_{2}, \ldots, x_{n}\right),\left(y_{1}, y_{2}, \ldots, y_{n}\right) \in X^{n}$ are two $Y$-coincidence points of $F$ and $g$, then

$$
g x_{i}=g y_{i} \quad \forall i .
$$

Let $\left(x_{1}, x_{2}, \ldots, x_{n}\right),\left(y_{1}, y_{2}, \ldots, y_{n}\right) \in X^{n}$ be two $\Upsilon$ coincidence points of $F$ and $g$, and let $\left(u_{1}, u_{2}, \ldots, u_{n}\right) \in X^{n}$ be a point such that $\left(g u_{1}, g u_{2}, \ldots, g u_{n}\right)$ is comparable, at the same time, to $\left(g x_{1}, g x_{2}, \ldots, g x_{n}\right)$ and to $\left(g y_{1}, g y_{2}, \ldots, g y_{n}\right)$. Using $\left(u_{1}, u_{2}, \ldots, u_{n}\right)$, define the following sequences. Let $u_{0}^{i}=u_{i}$ for all $i$. Reasoning as in Theorem 24, we can determine sequences $\left\{u_{m}^{1}\right\}_{m \geq 0},\left\{u_{m}^{2}\right\}_{m \geq 0}, \ldots,\left\{u_{m}^{n}\right\}_{m \geq 0}$ such that $g u_{m+1}^{i}=F\left(u_{m}^{\sigma_{i}(1)}, u_{m}^{\sigma_{i}(2)}, \ldots, u_{m}^{\sigma_{i}(n)}\right)$ for all $m$ and all $i$. We are going to prove that $g x_{i}=\lim _{m \rightarrow 0}^{d_{p}} g u_{m}^{i}=g y_{i}$ for all $i$, so (59) will be true.

Firstly, we reason with $\left(g u_{1}, g u_{2}, \ldots, g u_{n}\right)$ and $\left(g x_{1}, g x_{2}, \ldots, g x_{n}\right)$, and the same argument holds for $\left(g u_{1}, g u_{2}, \ldots, g u_{n}\right)$ and $\left(g y_{1}, g y_{2}, \ldots, g y_{n}\right)$. As $\left(g u_{1}, g u_{2}, \ldots, g u_{n}\right)$ and $\left(g x_{1}, g x_{2}, \ldots, g x_{n}\right)$ are comparable, we can suppose that $\left(g u_{1}, g u_{2}, \ldots, g u_{n}\right) \leq$ $\left(g x_{1}, g x_{2}, \ldots, g x_{n}\right)$ (the other case is similar); that is, $g u_{0}^{i}=g u_{i} \leq_{i} g x_{i}$ for all $i$. Using that $F$ has the mixed $g$-monotone property and reasoning as in Theorem 24 , it is possible to prove that $g u_{m}^{i} \leq_{i} g x_{i}$ for all $m \geq 1$ and all $i$. This condition implies that, for all $j$ and all $m \geq 1$

$$
\text { either }\left[g u_{m}^{\sigma_{j}(i)} \leq_{i} g x_{\sigma_{j}(i)} \forall i\right] \text { or }\left[g x_{\sigma_{j}(i)} \leq_{i} g u_{m}^{\sigma_{j}^{(i)}} \forall i\right] \text {. }
$$

$\left\{\beta_{m}\right\}_{m \geq 1} \rightarrow 0$ which means that $\lim _{m \rightarrow \infty} \beta_{m}=$ $\lim _{m \rightarrow \infty}\left(\max _{1 \leq i \leq n} p\left(g u_{m}^{i}, g x_{i}\right)\right)=0$. As $0 \leq p\left(g u_{m}^{i}, g x_{i}\right) \leq$ $\beta_{m}$ for all $m$ and all $i$, we deduce that $\left\{p\left(g u_{m}^{i}, g x_{i}\right)\right\}_{m \geq 1} \rightarrow$ $0=p\left(g x_{i}, g x_{i}\right)$ for all $i$; that is, $\left\{g u_{m}^{i}\right\} \stackrel{p}{\rightarrow} g x_{i}$ for all $i$ Item 1 of Lemma 20 shows that

$$
\left\{g u_{m}^{i}\right\} \stackrel{d_{p}}{\longrightarrow} g x_{i} \quad \forall i
$$

If we had supposed that $\left(g x_{1}, g x_{2}, \ldots, g x_{n}\right) \leq\left(g u_{1}, g u_{2}\right.$, $\left.\ldots, g u_{n}\right)$, we would have obtained the same property (61). And as $\left(g u_{1}, g u_{2}, \ldots, g u_{n}\right)$ also is comparable to $\left(g y_{1}, g y_{2}, \ldots, g y_{n}\right)$, we can reason in the same way to prove that $\left\{g u_{m}^{i}\right\} \stackrel{d_{p}}{\longrightarrow} g y_{i}$ for all $i$. Since the limit in a MS is unique, $g x_{i}=g y_{i}$ for all $i$.

Let $\left(x_{1}, x_{2}, \ldots, x_{n}\right) \in X^{n}$ be a $\Upsilon$-coincidence point of $F$ and $g$, and define $z_{i}=g x_{i}$ for all $i$. As $\left(z_{1}, z_{2}, \ldots, z_{n}\right)=$ $\left(g x_{1}, g x_{2}, \ldots, g x_{n}\right)$, Remark 6 assures us that $\left(z_{1}, z_{2}, \ldots, z_{n}\right)$ also is a $Y$-coincidence point of $F$ and $g$.

Step 2. We claim that $\left(z_{1}, z_{2}, \ldots, z_{n}\right)$ is the unique $\Upsilon$ coincidence point of $F$ and $g$ such that $g z_{i}=z_{i}$ for all $i$. It is similar to Step 2 in Theorem 11 in [11].

It is natural to say that $g$ is injective on the set of all $\Upsilon$ coincidence points of $F$ and $g$ when $g x_{i}=g y_{i}$ for all $i$ implies $x_{i}=y_{i}$ for all $i$ when $\left(x_{1}, x_{2}, \ldots, x_{n}\right),\left(y_{1}, y_{2}, \ldots, y_{n}\right) \in X^{n}$ are two $Y$-coincidence points of $F$ and $g$. For example, this is true is $g$ is injective on $X$.

Corollary 31. In addition to the hypotheses of Theorem 30, suppose that $g$ is injective on the set of all $\Upsilon$-coincidence points of $F$ and $g$. Then, $F$ and $g$ have a unique $Y$-coincidence point.

Proof. If $\left(x_{1}, x_{2}, \ldots, x_{n}\right)$ and $\left(y_{1}, y_{2}, \ldots, y_{n}\right)$ are two $\Upsilon$ coincidence points of $F$ and $g$, we have proved in (59) that $g x_{i}=g y_{i}$ for all $i$. As $g$ is injective on these points, then, $x_{i}=y_{i}$ for all $i$.

Corollary 32. In addition to the hypotheses of Theorem 30, suppose that $\left(z_{\sigma_{i}(1)}, z_{\sigma_{i}(2)}, \ldots, z_{\sigma_{i}(n)}\right)$ is comparable to $\left(z_{\sigma_{j}(1)}, z_{\sigma_{j}(2)}, \ldots, z_{\sigma_{j}(n)}\right)$ for all $i, j$. Then, $z_{1}=z_{2}=\cdots=z_{n}$.

In particular, there exists a unique $z \in X$ such that $F(z, z, \ldots, z)=z$, which verifies $g z=z$.

Proof. Let $M=\max _{1 \leq i, j \leq n} p\left(z_{i}, z_{j}\right)$, let $j_{0}, s_{0} \in\{1,2, \ldots, n\}$ such that $p\left(z_{j_{0}}, z_{s_{0}}\right)=M$, and let

$$
\Lambda=\max _{1 \leq i \leq n} p\left(z_{\sigma_{j_{0}}(i)}, z_{\sigma_{s_{0}}(i)}\right) \leq M
$$

Define $\beta_{m}=\max _{1 \leq i \leq n} p\left(g u_{m}^{i}, g x_{i}\right)$ for all $m$. Reasoning as in Theorem 24, it is not difficult to prove that 
Fix $j, s \in\{1,2, \ldots, n\}$. As $\left(z_{\sigma_{j}(1)}, z_{\sigma_{j}(2)}, \ldots, z_{\sigma_{j}(n)}\right)$ is comparable to $\left(z_{\sigma_{s}(1)}, z_{\sigma_{s}(2)}, \ldots, z_{\sigma_{s}(n)}\right)$, then either $z_{\sigma_{j}(i)} \leq_{i} z_{\sigma_{s}(i)}$ for all $i$ or $z_{\sigma_{s}(i)} \leq_{i} z_{\sigma_{j}(i)}$ for all $i$. Since $g z_{i}=z_{i}$ for all $i$, we know that either $g z_{\sigma_{j}(i)} \leq_{i} g z_{\sigma_{s}(i)}$ for all $i$ or $g z_{\sigma_{s}(i)} \leq_{i} g z_{\sigma_{j}(i)}$ for all $i$. In any case, applying (17),

$$
\begin{aligned}
\psi(M)= & \psi\left(p\left(z_{j_{0}}, z_{s_{0}}\right)\right) \\
= & \psi\left(p\left(g z_{j_{0}}, g z_{s_{0}}\right)\right) \\
= & \psi\left(p \left(F\left(z_{\sigma_{j_{0}}(1)}, z_{\sigma_{j_{0}}(2)}, \ldots, z_{\sigma_{j_{0}}(n)}\right),\right.\right. \\
& \left.\left.F\left(z_{\sigma_{s_{0}}(1)}, z_{\sigma_{s_{0}}(2)}, \ldots, z_{\sigma_{s_{0}}(n)}\right)\right)\right) \\
\leq & \psi\left(\max _{1 \leq i \leq n} p\left(g z_{\sigma_{j_{0}}(i)}, g z_{\sigma_{s_{0}}(i)}\right)\right) \\
& -\varphi\left(\max _{1 \leq i \leq n} p\left(g z_{\sigma_{j_{0}}(i)}, g z_{\sigma_{s_{0}}}(i)\right)\right) \\
= & \psi(\Lambda)-\varphi(\Lambda) \leq \psi(M)-\varphi(\Lambda) .
\end{aligned}
$$

If $\Lambda>0$, then $\varphi(\Lambda)>0$, so $\psi(M) \leq \psi(M)-\varphi(\Lambda)<\psi(M)$, which is impossible. Then, $\Lambda=0$, and (63) implies that $\psi(M) \leq \psi(\Lambda)-\varphi(\Lambda)=\psi(0)-\varphi(0)=0$, so $\psi(M)=0$. Therefore, $p\left(z_{i}, z_{j}\right)=0$ for all $i$ and $j$.

Example 33. Let $X=\mathbb{R}$ provided with its usual partial order $\leq$ and the partial metric $p(x, y)=\max (|x|,|y|)$. Let $n \in \mathbb{N}$, and let $a_{1}, a_{2}, \ldots, a_{n} \in \mathbb{R} \backslash\{0\}$ real numbers such that there exist $i_{0}, j_{0} \in\{1,2, \ldots, n\}$ verifying $a_{i_{0}}<0<a_{j_{0}}$. Let $N>\left|a_{1}\right|+$ $\left|a_{2}\right|+\cdots+\left|a_{n}\right|$, and consider $F\left(x_{1}, x_{2}, \ldots, x_{n}\right)=\left(a_{1} x_{1}+a_{2} x_{2}+\right.$ $\left.\cdots+a_{n} x_{n}\right) / N$ and $g x=x$, for all $x, x_{1}, x_{2}, \ldots, x_{n} \in X$. Then, $F$ is monotone nondecreasing in those arguments for which $a_{i}>0$ and monotone nonincreasing in those arguments for which $a_{i}<0$. Furthermore, taking $k=\left(\left|a_{1}\right|+\left|a_{2}\right|+\cdots+\right.$ $\left.\left|a_{n}\right|\right) / N \in(0,1)$, it follows that

$$
\begin{aligned}
& \left|F\left(x_{1}, x_{2}, \ldots, x_{n}\right)\right| \\
& \quad \leq \frac{\left|a_{1}\right|\left|x_{1}\right|+\left|a_{2}\right|\left|x_{2}\right|+\cdots+\left|a_{n}\right|\left|x_{n}\right|}{N} \\
& \quad \leq \frac{\left|a_{1}\right|+\left|a_{2}\right|+\cdots+\left|a_{n}\right|}{N} \max \left(\left|x_{1}\right|,\left|x_{2}\right|, \ldots,\left|x_{n}\right|\right) \\
& \quad=k \max \left(\left|x_{1}\right|,\left|x_{2}\right|, \ldots,\left|x_{n}\right|\right) .
\end{aligned}
$$

Therefore,

$$
\begin{aligned}
& p\left(F\left(x_{1}, x_{2}, \ldots, x_{n}\right), F\left(y_{1}, y_{2}, \ldots, y_{n}\right)\right) \\
& =\max \left(\left|F\left(x_{1}, x_{2}, \ldots, x_{n}\right)\right|,\left|F\left(y_{1}, y_{2}, \ldots, y_{n}\right)\right|\right) \\
& \leq \max \left(k \max \left(\left|x_{1}\right|,\left|x_{2}\right|, \ldots,\left|x_{n}\right|\right),\right. \\
& \left.\quad k \max \left(\left|y_{1}\right|,\left|y_{2}\right|, \ldots,\left|y_{n}\right|\right)\right) \\
& =k \max _{i}\left(p\left(x_{i}, y_{i}\right)\right) .
\end{aligned}
$$

If $\psi(t)=t$ and $\varphi(t)=(1-k) t$, all conditions of Theorems 24 and 30 (and Corollaries 31 and 32 ) are satisfied. Indeed, it is clear that $(0,0, \ldots, 0)$ is the unique fixed point of $F$.

The following example is based on Examples 1.9 and 2.2 in [29].

Example 34. Let $X=\{0,1,2,3,4\}$, and let $p$ be the partial metric on $X$ given by $p(x, y)=\max (x, y)$ for all $x, y \in$ $X$. Then, $(X, p)$ is complete, and $p$ generates the discrete topology on $X$ (indeed, $d_{p}$ is the Euclidean metric on $X$ ). Consider on $X$ the following partial order:

$$
x, y \in X, \quad x \leq y \Longleftrightarrow x=y \quad \text { or } \quad(x, y)=(0,2) \text {. }
$$

Consider $F: X^{n} \rightarrow X$ and $g: X \rightarrow X$ defined by

$$
\begin{gathered}
F\left(x_{1}, x_{2}, \ldots, x_{n}\right)= \begin{cases}0, & \text { if } x_{1}, x_{2}, \ldots, x_{n} \in\{0,1,2\} \\
1, & \text { otherwise, }\end{cases} \\
g x= \begin{cases}0, & \text { if } x=0, \\
2, & \text { if } x \in\{0.5,1\} \\
3, & \text { if } x \in\{1.5,2\} .\end{cases}
\end{gathered}
$$

It is not difficult to prove the following statements.

(1) $F$ and $g$ are $\alpha_{p}$-continuous mappings (since $d_{p}$ generates the discrete topology on $X$ ).

(2) $F$ and $g$ are commuting.

(3) If $y, z \in X$ verify $g y \leq g z$, then either $y, z \in$ $\{0,1,2\}$ or $y, z \in\{3,4\}$. Then, $F$ has the mixed $(g, \leq)$ monotone property on $X$.

(4) If $x_{1}, x_{2}, \ldots, x_{n}, y_{1}, y_{2}, \ldots, y_{n} \in X$ verify $g x_{i} \leq_{i} g y_{i}$ for all $i$, then $F\left(x_{1}, x_{2}, \ldots, x_{n}\right)=F\left(y_{1}, y_{2}, \ldots, y_{n}\right)$. In particular, (17) holds (whatever $\psi$ and $\varphi$; for instance, $\psi(t)=2 t$ and $\varphi(t)=\log (t+1)$ for all $t \geq 0)$.

For simplicity, henceforth, suppose that $n$ is even, and let $A$ (resp., $B$ ) be the set of all odd (resp., even) numbers in $\{1,2, \ldots, n\}$.

(5) For a mapping $\sigma: \Lambda_{n} \rightarrow \Lambda_{n}$, we use the notation $\sigma \equiv(\sigma(1), \sigma(2), \ldots, \sigma(n))$ and consider

$\sigma_{i} \equiv(i, i+1, \ldots, n-1, n, 1,2, \ldots, i-1) \quad \forall i$.

Then, $\sigma_{i} \in \Omega_{A, B}$ if $i$ is odd, and $\sigma_{i} \in \Omega_{A, B}^{\prime}$ if $i$ is even. Let $\Upsilon=\left(\sigma_{1}, \sigma_{2}, \ldots, \sigma_{n}\right)$.

(6) Take $x_{0}^{i}=0$ if $i$ is odd and $x_{0}^{i}=2$ if $i$ is even. Then, $g x_{0}^{i} \leq_{i} F\left(x_{0}^{\sigma_{i}(1)}, x_{0}^{\sigma_{i}(2)}, \ldots, x_{0}^{\sigma_{i}(n)}\right)$ for all $i$.

(7) $\left(X, d_{p}, \leq\right)$ has the sequential $g$-monotone property.

Therefore, we can apply Theorems 24 and 30, and Corollaries 31 and 32 , to conclude that $F$ and $g$ have a unique $\Upsilon$ coincidence point, which is $(0,0, \ldots, 0)$. 


\section{Acknowledgments}

This work has been partially supported by Junta de Andaluca, by projects FQM-268, FQM-178 and FQM-235 of the Andalusian CICYE.

\section{References}

[1] D. J. Guo and V. Lakshmikantham, "Coupled fixed points of nonlinear operators with applications," Nonlinear Analysis: Theory, Methods \& Applications, vol. 11, no. 5, pp. 623-632, 1987.

[2] T. Gnana Bhaskar and V. Lakshmikantham, "Fixed point theorems in partially ordered metric spaces and applications," Nonlinear Analysis: Theory, Methods \& Applications, vol. 65, no. 7, pp. 1379-1393, 2006.

[3] J.-X. Fang, "Common fixed point theorems of compatible and weakly compatible maps in Menger spaces," Nonlinear Analysis: Theory, Methods \& Applications, vol. 71, no. 5-6, pp. 1833-1843, 2009.

[4] X.-Q. Hu, "Common coupled fixed point theorems for contractive mappings in fuzzy metric spaces," Fixed Point Theory and Applications, Article ID 363716, 14 pages, 2011.

[5] V. Lakshmikantham and L. Ćirić, "Coupled fixed point theorems for nonlinear contractions in partially ordered metric spaces," Nonlinear Analysis: Theory, Methods \& Applications, vol. 70, no. 12, pp. 4341-4349, 2009.

[6] S. Shakeri, L. J. B. Ćirić, and R. Saadati, "Common fixed point theorem in partially ordered $L$-fuzzy metric spaces," Fixed Point Theory and Applications, vol. 2010, Article ID 125082, 13 pages, 2010.

[7] V. Berinde and M. Borcut, "Tripled fixed point theorems for contractive type mappings in partially ordered metric spaces," Nonlinear Analysis: Theory, Methods \& Applications, vol. 74, no. 15, pp. 4889-4897, 2011.

[8] V. Berinde, "Approximating common fixed points of noncommuting almost contractions in metric spaces," Fixed Point Theory, vol. 11, no. 2, pp. 179-188, 2010.

[9] M. Borcut and V. Berinde, "Tripled coincidence theorems for contractive type mappings in partially ordered metric spaces," Applied Mathematics and Computation, vol. 218, no. 10, pp. 5929-5936, 2012.

[10] M. Turinici, "Product fixed points in ordered metric spaces," http://arxiv.org/abs/1110.3079 .

[11] A. Roldán, J. Martínez-Moreno, and C. Roldán, "Multidimensional fixed point theorems in partially ordered complete metric spaces," Journal of Mathematical Analysis and Applications, vol. 396, no. 2, pp. 536-545, 2012.

[12] M. Berzig and B. Samet, "An extension of coupled fixed point's concept in higher dimension and applications," Computers \& Mathematics with Applications, vol. 63, no. 8, pp. 1319-1334, 2012.

[13] E. Karapınar and V. Berinde, "Quadruple fixed point theorems for nonlinear contractions in partially ordered metric spaces," Banach Journal of Mathematical Analysis, vol. 6, no. 1, pp. 7489, 2012.

[14] S. G. Matthews, "Partial metric topology, general topology and its applications," in Proceedings of the 8th Summer Conference, Queen's College, vol. 728, pp. 183-197, Annals of the New York Academy of Sciences, 1994.

[15] İ. M. Erhan, E. Karapınar, and A. Öztürk, "Fixed point theorems on quasi-partial metric spaces," Mathematical and Computer Modelling, vol. 57, no. 9-10, pp. 2442-2448, 2013.
[16] W. Shatanawi, B. Samet, and M. Abbas, "Coupled fixed point theorems for mixed monotone mappings in ordered partial metric spaces," Mathematical and Computer Modelling, vol. 55, no. 3-4, pp. 680-687, 2012.

[17] H. Aydi, E. Karapınar, and W. Shatanawi, "Coupled fixed point results for $(\psi, \varphi)$-weakly contractive condition in ordered partial metric spaces," Computers \& Mathematics with Applications, vol. 62, no. 12, pp. 4449-4460, 2011.

[18] B. S. Choudhury, N. Metiya, and A. Kundu, "Coupled coincidence point theorems in ordered metric spaces," Annali dell'Universitá di Ferrara, vol. 57, no. 1, pp. 1-16, 2011.

[19] E. Karapınar and N. V. Luong, "Quadruple fixed point theorems for nonlinear contractions," Computers \& Mathematics with Applications, vol. 64, no. 6, pp. 1839-1848, 2012.

[20] N. V. Luong and N. X. Thuan, "Coupled fixed points in partially ordered metric spaces and application," Nonlinear Analysis: Theory, Methods \& Applications, vol. 74, no. 3, pp. 983-992, 2011.

[21] R. H. Haghi, Sh. Rezapour, and N. Shahzad, "Be careful on partial metric fixed point results," Topology and its Applications, vol. 160, no. 3, pp. 450-454, 2013.

[22] L. Ćirić, M. Abbas, B. Damjanović, and R. Saadati, "Common fuzzy fixed point theorems in ordered metric spaces," Mathematical and Computer Modelling, vol. 53, no. 9-10, pp. 1737-1741, 2011.

[23] N. Shobkolaei, S. M. Vaezpour, and S. Sedghi, "A common fixed point theorem on ordered partial metric spaces," Journal of Applied Sciences Research, vol. 1, pp. 3433-3439, 2011.

[24] E. Karapınar, N. Shobkolaei, S. Sedghi, and S. M. Vaezpour, "A common fixed point theorem for cyclic operators on partial metric spaces," Filomat, vol. 26, pp. 407-414, 2012.

[25] E. Karapınar and İ. M. Erhan, "Fixed point theorems for operators on partial metric spaces," Applied Mathematics Letters, vol. 24, no. 11, pp. 1894-1899, 2011.

[26] T. Abdeljawad, E. Karapınar, and K. Taş, "Existence and uniqueness of a common fixed point on partial metric spaces," Applied Mathematics Letters, vol. 24, no. 11, pp. 1900-1904, 2011.

[27] M. S. Khan, M. Swaleh, and S. Sessa, "Fixed point theorems by altering distances between the points," Bulletin of the Australian Mathematical Society, vol. 30, no. 1, pp. 1-9, 1984.

[28] A. Roldán, J. Martínez-Moreno, C. Roldán, and E. Karapınar, "Meir-Keeler type multidimensional fixed point theorems in partially ordered metric spaces," Abstract and Applied Analysis, vol. 2013, Article ID 406026, 9 pages, 2013.

[29] N. M. Hung, E. Karapinar, and N. V. Luong, "Coupled coincidence point theorem in partially ordered metric spaces via implicit relation," Abstract and Applied Analysis, vol. 2012, Article ID 796964, 14 pages, 2012. 


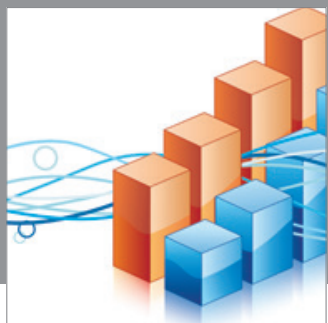

Advances in

Operations Research

mansans

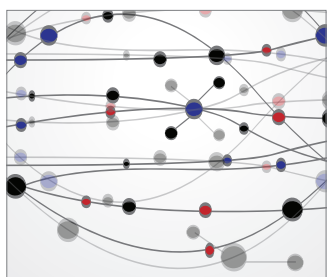

The Scientific World Journal
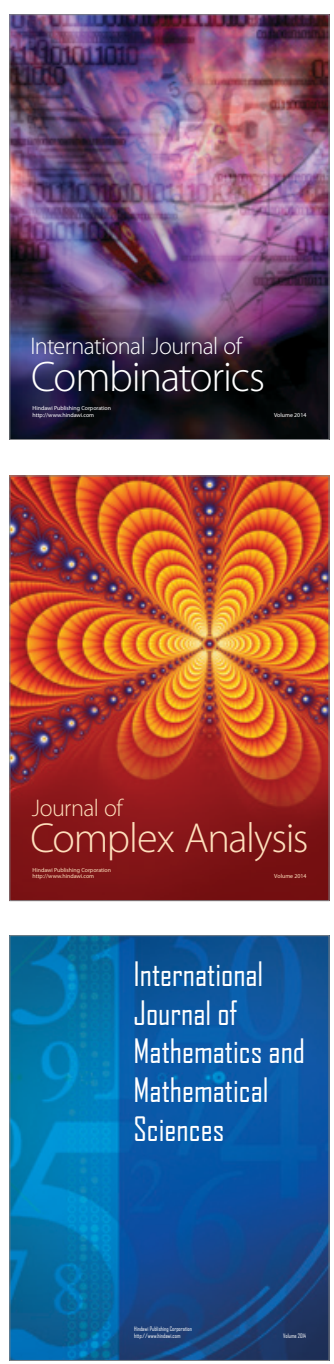
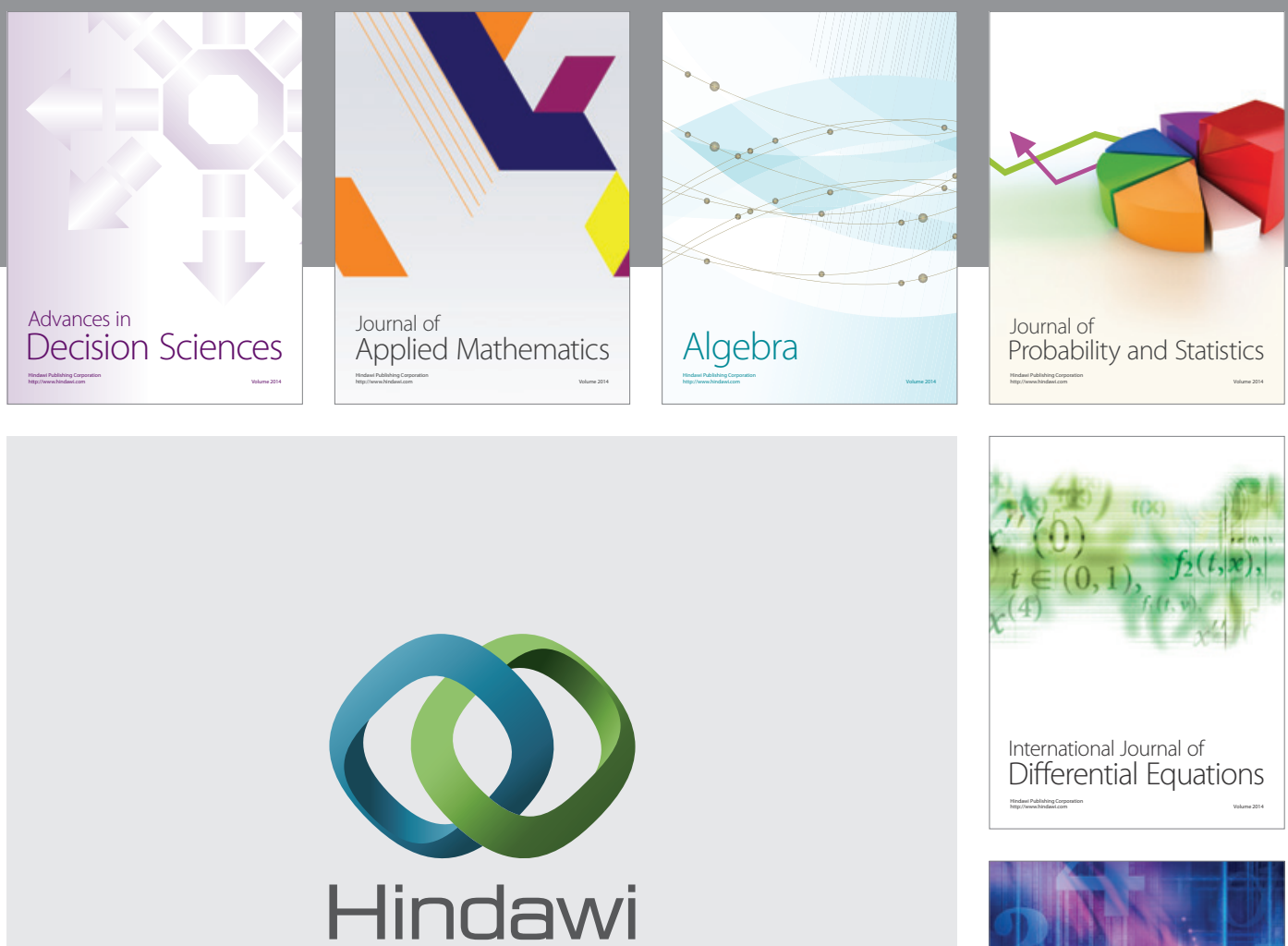

Submit your manuscripts at http://www.hindawi.com
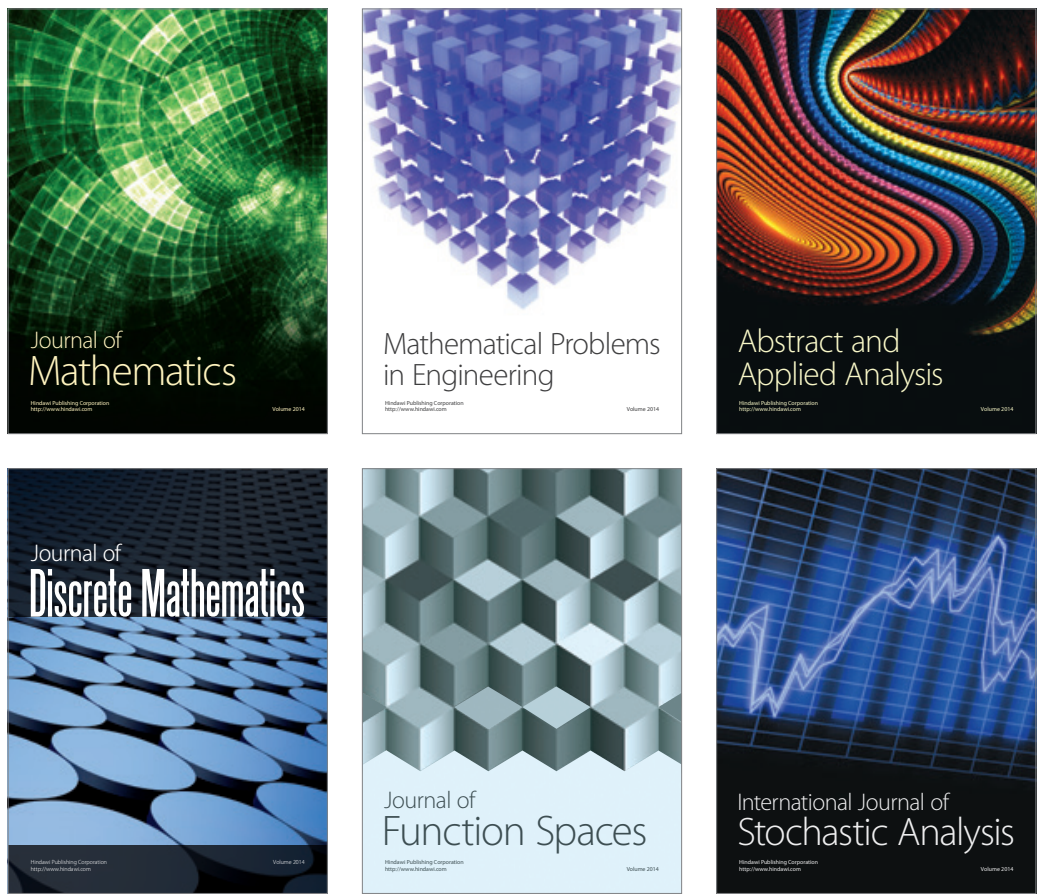

Journal of

Function Spaces

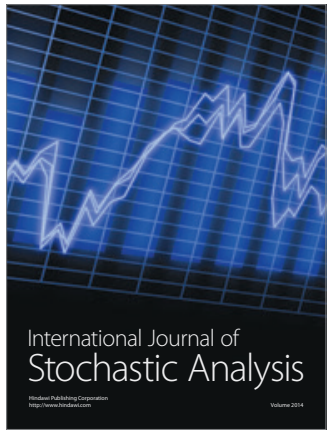

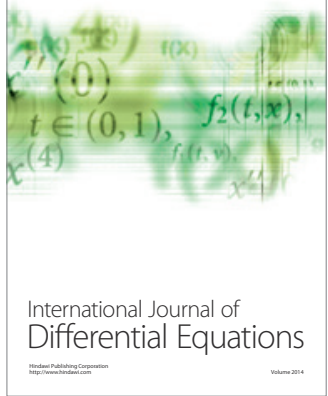
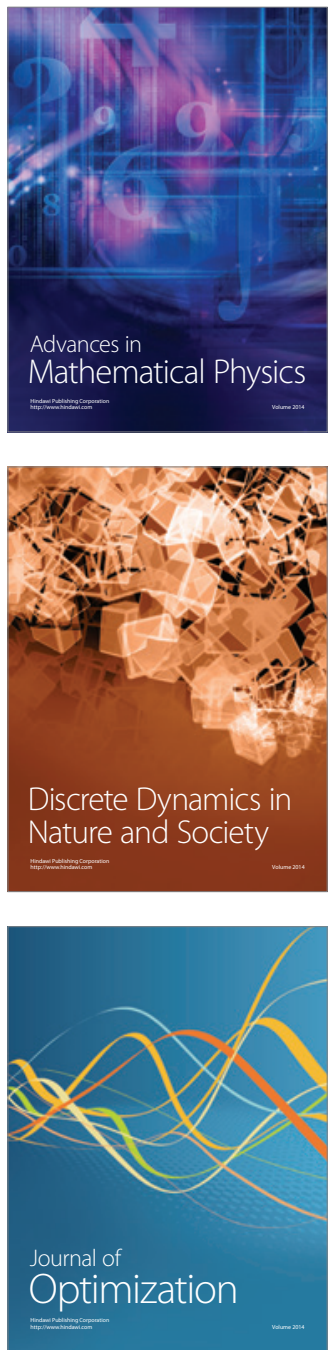